Pacific

Journal of

Mathematics

REPRESENTATION THEORY OF GL( $n$ ) OVER A $p$-ADIC DIVISION ALGEBRA AND UNITARITY IN THE JACQUET-LANGLANDS CORRESPONDENCE

MARKO TADIĆ 


\title{
REPRESENTATION THEORY OF GL(n) OVER A $p$-ADIC DIVISION ALGEBRA AND UNITARITY IN THE JACQUET-LANGLANDS CORRESPONDENCE
}

\author{
MARKO TADIĆ
}

\begin{abstract}
Let $F$ be a local nonarchimedean field of characteristic 0 , and let $A$ be an $F$-central division algebra of dimension $d_{A}$ over $F$. In this paper, we first develop some parts of the representation theory of $\operatorname{GL}(m, A)$, assuming the conjecture that unitary parabolic induction is irreducible for $\operatorname{GL}(m, A)$ 's. Among others, we obtain the formula for characters of irreducible unitary representations of $\operatorname{GL}(m, A)$ in terms of standard characters. Then we study the Jacquet-Langlands correspondence on the level of Grothendieck groups of $\operatorname{GL}\left(p d_{A}, F\right)$ and $\operatorname{GL}(p, A)$. Using this character formula, we get explicit formulas for the Jacquet-Langlands correspondence of irreducible unitary representations of $\operatorname{GL}(n, F)$ (assuming the conjecture to hold). As a consequence, we get that the Jacquet-Langlands correspondence sends irreducible unitary representations of $\operatorname{GL}(n, F)$ either to zero or to irreducible unitary representations, up to a sign.
\end{abstract}

\section{Introduction}

Functoriality is a key aspect of the Langlands program [1970]. One of the first examples of functoriality which were studied in the local case was the connection between representations of various inner forms of GL( $n)$ (see [Knapp and Rogawski 1997]). The first example, studied already in [Jacquet and Langlands 1970], was the connection between irreducible representations of GL(2) over a local field $F$ and irreducible representations of the multiplicative group of the quaternion algebra over $F$. (The $L$ groups of these two groups are both $\operatorname{GL}(2, \mathbb{C}) \times \operatorname{Gal}(\bar{F} / F)$, and the functoriality considered here corresponds to the identity mapping.)

Let $F$ be a local nonarchimedean field of characteristic 0 and let $A$ be an $F$ central division algebra of rank $d_{A}$ over $F$. For each positive integer $p$, P. Deligne,

MSC2000: 22E50, 22E35, 11F70, 11S37.

Keywords: local nonarchimedean field, division algebra, general linear group, Jacquet-Langlands correspodence, functoriality.

The author was partly supported by Croatian Ministry of Science and Technology grant \#37108. 
D. Kazhdan and M.-F. Vignéras established bijections

$$
\mathrm{LJ}_{p d_{A}}
$$

between irreducible essentially square-integrable representations of $\operatorname{GL}\left(p d_{A}, F\right)$ and $\operatorname{GL}(p, A)$ ([Rogawski 1983] takes care of the case $p=1$ ). The crucial requirement which holds for these bijections, and which characterizes them uniquely, is that the characters $\Theta_{\delta}$ and $\Theta_{\mathrm{LJ}_{p d_{A}}(\delta)}$ of the representations $\delta$ and $\mathrm{LJ}_{p d_{A}}(\delta)$ satisfy the character identity

$$
(-1)^{p d_{A}} \Theta_{\delta}(g)=(-1)^{p} \Theta_{\mathrm{LJ}_{p d_{A}}(\delta)}\left(g^{\prime}\right)
$$

whenever $g$ and $g^{\prime}$ have the same characteristic polynomials, and when this polynomial is separable. These bijections are called Jacquet-Langlands correspondences.

A. I. Badulescu observed that Jacquet-Langlands correspondences extend in a very natural way to mappings between Grothendieck groups

$$
\mathrm{LJ}_{p d_{A}}: \text { Groth GL }\left(p d_{A}, F\right) \rightarrow \operatorname{Groth} \operatorname{GL}(p, A),
$$

such that the extensions are compatible with parabolic induction, i.e. that they commute with parabolic induction. (Essentially, such extensions are unique if we require that characters of $\operatorname{GL}(n, F)$ 's go to 0 if $d_{A} \nmid n$.) Moreover, these extensions satisfy the character identity above on the level of formal characters (for a precise description of the extensions, see $\S 6.1$ ). We shall call these mappings JacquetLanglands correspondences on the level of Grothendieck groups.

For the group $G$ of rational points of a reductive group defined over a local nonarchimedean field, we denote by $\tilde{G}$ the set of all the equivalence classes of irreducible smooth representations of $G$. The unitary dual $\hat{G}$ of $G$ consists of all the unitarizable classes in $\tilde{G}$.

We consider $\operatorname{GL}(n, F)^{\sim}$ as a subset of $\operatorname{Groth} \operatorname{GL}(n, F)$ in a natural way (it forms a $\mathbb{Z}$-basis). An interesting question is to understand what happens with irreducible representations under the Jacquet-Langlands correspondence on the level of Grothendieck groups, and in particular, what happens with irreducible unitary representations. Already, very simple examples will show that $\mathrm{LJ}_{n}$ will carry some irreducible representations to zero. Further, it is not hard to see that an irreducible (unitarizable) representation can go to the negative of an irreducible representation.

In this paper we study what happens with irreducible unitary representations under the Jacquet-Langlands correspondence, assuming the following conjecture for general linear groups over division algebras, introduced in [Tadić 1990]:

$$
\text { unitary parabolic induction is irreducible for } \operatorname{GL}(m, A) \text { 's. }
$$


In other words, if $\pi_{1}$ and $\pi_{2}$ are irreducible unitary representations of $\operatorname{GL}\left(m_{1}, A\right)$ and $\operatorname{GL}\left(m_{2}, A\right)$, the parabolically induced representation $\operatorname{Ind}^{\mathrm{GL}\left(m_{1}+m_{2}, A\right)}\left(\pi_{1} \otimes \pi_{2}\right)$ is irreducible.

Note that [Vogan 1986] implies that (U0) holds in the archimedean case. Also, J. Bernstein [1984] proved that (U0) holds if $A=F$ (unfortunately his method cannot be extended to the division algebra case).

Here we first develop some directions of the representation theory of GL(n) over division algebras over a local nonarchimedean field, to be able to obtain the formula for characters of irreducible unitary representations of $\mathrm{GL}(m, A)$ in terms of standard characters. (Assuming (U0) to hold, [Tadić 1990] and [Badulescu and Renard 2004] imply a classification of the unitary duals $\operatorname{GL}(m, A)^{\wedge}$ of $\operatorname{GL}(m, A)$ for all $m \geq 1$.) Using this character formula, we compute explicit formulas for $\mathrm{LJ}_{n}(\pi), \pi \in \mathrm{GL}(n, F)^{\wedge}$ (Propositions 7.3, 9.5 and Section 11). As a consequence:

Corollary. Assume that (U0) holds. Then

$$
\mathrm{LJ}_{p d_{A}}\left(\mathrm{GL}\left(p d_{A}, F\right)^{\wedge}\right) \subseteq \pm \mathrm{GL}(p, A)^{\wedge} \cup\{0\} ;
$$

that is, Jacquet-Langlands correspondences send irreducible unitary representations of general linear groups over $F$ either again to irreducible unitary representations of general linear groups over A, up to a sign, or to 0 .

Remark. The following direct consequence may be of some interest. Let $\sigma$ be an element of $\operatorname{GL}(p, A)^{\sim}$ (resp. of (Groth $\left.\left.\operatorname{GL}(p, A)\right) \backslash\{0\}\right)$. Suppose that (U0) holds and suppose that there exists $\pi \in \mathrm{GL}\left(p d_{A}, F\right)^{\wedge}$ such that the characters of $\sigma$ and $\pi$ are equal up to (the same) sign on elements with same characteristic polynomials. Then $\sigma$ is unitarizable (resp. $\left.\sigma \in \pm \operatorname{GL}(p, A)^{\wedge}\right)$.

There are very strong formal similarities between the Jacquet-Langlands correspondences studied in this paper and the Kazhdan-Patterson lifting studied in [Tadić 1996].

We now give a description of the content of the paper according to sections. Section 1 goes over notation and necessary basic results for general linear groups over a local nonarchimedean field. Section 2 introduces notation and basic results for general linear groups over division algebras. In Section 3 we show that the canonical involution on irreducible representations of $\operatorname{GL}(m, A)$ (introduced by A.-M. Aubert, and by P. Schneider and U. Stuhler) preserves unitarity. We also obtain an explicit formula for the involution on irreducible unitary representations. (We assume throughout that (U0) holds.) In Section 4 we describe irreducible subquotients of ends of complementary series, obtaining in this way a character identity, which enable us to compute in Section 5 characters of irreducible unitary representations of $\mathrm{GL}(m, A)$ in terms of standard characters. In Section 6 we recall the Jacquet-Langlands correspondence on the level of Grothendieck groups. In 
Section 7 we compute $\mathrm{LJ}(\pi)$ for one of four basic types of $\pi \in \mathrm{GL}(n, F)^{\wedge}$, while the unitarity of $\operatorname{LJ}(\pi)$ is shown in Section 8. Sections 9 and 10 study the same problem for the second basic type of $\pi$. In Section 11 we compute the Jacquet-Langlands correspondence of the remaining two basic types of $\pi$, using canonical involutions.

\section{Some facts from the representation theory of $\operatorname{GL}(n, F)$}

We introduce the notation and basic results that we shall need for general linear groups over a local nonarchimedean field.

1.1. We fix a local nonarchimedean field $F$. The modulus character of $F$ will be denoted by ||$_{F}$ (it satisfies $|x|_{F} \int_{F} f(x a) d a=\int_{F} f(a) d a$ for any continuous compactly supported complex-valued function $f$ on $F$, where $d a$ denotes a Haar measure of the additive group $(F,+)$ of the field).

1.2. Let $G$ be the group of rational points of a reductive group over $F$. The set of equivalence classes of irreducible smooth representations of $G$ will be denoted by

$$
\tilde{G} .
$$

The subset of unitarizable classes in $\tilde{G}$ will be denoted by

$$
\hat{G} \text {. }
$$

A representation $\pi \in \tilde{G}$ is called essentially square-integrable if there exists a character $\chi$ of $G$ such that $\chi \pi$ is square-integrable representation modulo the center. All the essentially square-integrable classes in $\tilde{G}$ will be denoted by

$$
\mathscr{D}(G) \text {. }
$$

The Grothendieck group of the category of all representations of $G$ of finite length will be denoted by

\section{Groth $G$.}

1.3. Now we introduce the Bernstein-Zelevinsky notation for the general linear group GL $(n, F)$ (for more on notation see [Zelevinsky 1980; Tadić 1986]).

For two smooth representations $\pi_{1}$ and $\pi_{2}$ of $\operatorname{GL}\left(n_{1}, F\right)$ and $\operatorname{GL}\left(n_{2}, F\right)$, we consider $\pi_{1} \otimes \pi_{2}$ as a representation of $\operatorname{GL}\left(n_{1}, F\right) \times \operatorname{GL}\left(n_{2}, F\right)$. Identifying in a natural way $\operatorname{GL}\left(n_{1}, F\right) \times \operatorname{GL}\left(n_{2}, F\right)$ with the Levi factor of the parabolic subgroup

$$
\left\{\left[\begin{array}{cc}
g_{1} & * \\
0 & g_{2}
\end{array}\right] ; g_{i} \in \mathrm{GL}\left(n_{i}, F\right) \text { for } i=1,2\right\},
$$

we denote by

$$
\pi_{1} \times \pi_{2}
$$


the smooth representation of GL $\left(n_{1}+n_{2}, F\right)$ parabolically induced by $\pi_{1} \otimes \pi_{2}$. Then

$$
\left(\pi_{1} \times \pi_{2}\right) \times \pi_{3} \cong \pi_{1} \times\left(\pi_{2} \times \pi_{3}\right) .
$$

1.4. The characters of $F^{\times}$will be identified with characters of $\operatorname{GL}(n, F)$ using the determinant homomorphism. The character of $\operatorname{GL}(n, F)$ corresponding to ||$_{F}$ will be denoted by

$$
\text { v. }
$$

For any character $\chi$ of $F^{\times}$,

$$
\chi\left(\pi_{1} \times \pi_{2}\right) \cong\left(\chi \pi_{1}\right) \times\left(\chi \pi_{2}\right) .
$$

\subsection{Let}

$$
R_{n, F}=\operatorname{Groth} \operatorname{GL}(n, F) .
$$

Then $\operatorname{GL}(n, F)^{\sim}$ is a $\mathbb{Z}$-basis of $R_{n, F}$.

We can lift $\times$ to a $\mathbb{Z}$-bilinear mapping $\times: R_{n_{1}, F} \times R_{n_{2}, F} \rightarrow R_{n_{1}+n_{2}, F}$ since the semisimplification of $\pi_{1} \times \pi_{2}$ depends only on semisimplifications of $\pi_{1}$ and $\pi_{2}$. Set

$$
R_{F}=\bigoplus_{n \geq 0} R_{n, F} .
$$

One extends $\times$ to an operation $\times: R_{F} \times R_{F} \rightarrow R_{F}$ in an obvious way, and $R_{F}$ becomes an associative, commutative graded ring.

Fix a character $\chi$ of $F^{\times}$. Lift the mappings $\pi \mapsto \chi \pi: R_{n, F} \rightarrow R_{n, F}$ to a $\mathbb{Z}$-linear map $\chi: R_{F} \rightarrow R_{F}$. In this way we get an endomorphism of the graded ring $R_{F}$.

We have a natural partial ordering on $R_{n, F}$. $\left(\operatorname{GL}(n, F)^{\sim}\right.$ generates the cone of positive elements in $R_{n, F}$.) Then orderings on the $R_{n, F}$ 's determine an ordering $\leq$ on $R_{F}$ in a natural way. An additive homomorphism $\phi: R_{F} \rightarrow R_{F}$ will be called positive if $x \in R_{F}, x \geq 0$ implies $\phi(x) \geq 0$.

\subsection{Set}

$$
\mathscr{D}_{F}=\bigcup_{n \geq 1} \mathscr{D}(\mathrm{GL}(n, F)) .
$$

For $\delta \in \mathscr{D}_{F}$ there exists a unique $e(\delta) \in \mathbb{R}$ such that $v^{-e(\delta)} \delta$ is unitarizable. The representation $v^{-e(\delta)} \delta$ will be denoted by $\delta^{u}$. In this way,

$$
\delta=v^{e(\delta)} \delta^{u},
$$

where $e(\delta) \in \mathbb{R}$ and $\delta^{u}$ is unitarizable.

1.7. We now describe the Langlands classification for general linear groups. Let $M\left(\mathscr{D}_{F}\right)$ be the set of all finite multisets in $\mathscr{D}_{F}$ and $d=\left(\delta_{1}, \delta_{2}, \ldots, \delta_{k}\right) \in M\left(\mathscr{D}_{F}\right)$. Let $\gamma$ be a permutation of $\{1,2, \ldots, k\}$ such that $e\left(\delta_{\gamma(1)}\right) \geq e\left(\delta_{\gamma(2)}\right) \geq \cdots \geq e\left(\delta_{\gamma(k)}\right)$. 
The representation

$$
\lambda(d)=\delta_{\gamma(1)} \times \delta_{\gamma(2)} \times \cdots \times \delta_{\gamma(k)}
$$

has a unique irreducible quotient. Its class depends only on $d$ (not on $\gamma$ as above). This unique irreducible quotient will be denoted by $L(d)$ or $L\left(\delta_{1}, \delta_{2}, \ldots, \delta_{k}\right)$. From $\S 1.4$ it follows that for a character $\chi$ of $F^{\times}$,

$$
\chi L\left(\delta_{1}, \delta_{2}, \ldots, \delta_{k}\right) \cong L\left(\chi \delta_{1}, \chi \delta_{2}, \ldots, \chi \delta_{k}\right) .
$$

To shorten the notation, we shall often denote the semisimplification of $\lambda(d)$ simply by $\lambda(d) \in R_{F}$. This will produce no confusion. From the properties of the Langlands classification it is well-known that the $\lambda(d) \in R_{F}, d \in M\left(\mathscr{D}_{F}\right)$, form a basis of $R_{F}$.

Proposition [Zelevinsky 1980]. The ring $R_{F}$ is a polynomial ring over $\mathscr{D}_{F}$.

1.8. One defines addition of elements of $M\left(\mathscr{D}_{F}\right)$ by

$$
\left(\delta_{1}, \delta_{2}, \ldots, \delta_{k}\right)+\left(\delta_{1}^{\prime}, \delta_{2}^{\prime}, \ldots, \delta_{k^{\prime}}^{\prime}\right)=\left(\delta_{1}, \delta_{2}, \ldots, \delta_{k}, \delta_{1}^{\prime}, \delta_{2}^{\prime}, \ldots, \delta_{k^{\prime}}^{\prime}\right) .
$$

Proposition [Rodier 1982]. For $d_{1}, d_{2} \in M\left(\mathscr{D}_{F}\right), L\left(d_{1}+d_{2}\right)$ is a subquotient of $L\left(d_{1}\right) \times L\left(d_{2}\right)$. The multiplicity is one.

1.9. Let $\mathscr{C}_{F}$ be the set of all the equivalence classes of irreducible cuspidal representations of all general linear groups $\operatorname{GL}(n, F), n \geq 1$. For $\rho \in \mathscr{C}_{F}$ and $k \in \mathbb{Z}, k \geq 0$, the set

$$
\left[\rho, v^{k} \rho\right]=\left\{\rho, v \rho, v^{2} \rho, \ldots, v^{k} \rho\right\}
$$

is called a segment of irreducible cuspidal representations. A segment of the form $\left[v^{k_{1}} \rho, v^{k_{2}} \rho\right.$ ] (where $k_{1}, k_{2} \in \mathbb{R}$ are such that $k_{2}-k_{1} \in \mathbb{Z}$ and $k_{2}-k_{1} \geq 0$ ) will also be denoted by

$$
\left[k_{1}, k_{2}\right]^{\rho} \text {. }
$$

The set of all such segments will be denoted by $\mathscr{Y}_{F}$. The set of all finite multisets in $\mathscr{S}_{F}$ will be denoted by $M\left(\mathscr{Y}_{F}\right)$. We consider the partial ordering $\leq$ on $M\left(\mathscr{Y}_{F}\right)$ introduced in [Zelevinsky 1980, §7.1], defined by linking segments.

1.10. Let $\Delta=\left[\rho, v^{k} \rho\right]=\left\{\rho, v \rho, v^{2} \rho, \ldots, v^{k} \rho\right\} \in \mathscr{Y}_{F}$. The representation

$$
\rho \times v \rho \times v^{2} \rho \times \cdots \times v^{k} \rho
$$

has a unique irreducible quotient, denoted by

$$
\delta\left(\left[\rho, v^{k} \rho\right]\right),
$$

and a unique irreducible subrepresentation, denoted by

$$
\delta\left(\left[\rho, v^{k} \rho\right]\right)^{t} .
$$


The representations $\delta\left(\left[\rho, v^{k} \rho\right]\right)$ are essentially square-integrable. Further, the representations $\delta\left(\left[\rho, v^{k} \rho\right]\right)^{t}$ are called Zelevinsky's segment representations.

By Bernstein-Zelevinsky theory, the mapping

$$
\delta: \mathscr{Y}_{F} \rightarrow \mathscr{D}_{F}, \quad \Delta \mapsto \delta(\Delta),
$$

is a bijection.

We can state this also in the following way. For $n \in \mathbb{N}$ and $\rho \in \mathscr{C}_{F}$, set

$$
\delta(\rho, n)=\delta\left([-(n-1) / 2,(n-1) / 2]^{\rho}\right) .
$$

Then $(\rho, n) \mapsto \delta(\rho, n)$ is a bijection of $\mathscr{C}_{F} \times \mathbb{N}$ onto $\mathscr{D}_{F}$.

We lift $\Delta \mapsto \delta(\Delta)$ naturally to a bijection

$$
M(\delta): M\left(\mathscr{Y}_{F}\right) \rightarrow M\left(\mathscr{D}_{F}\right), \quad\left(\Delta_{1}, \ldots, \Delta_{k}\right) \mapsto\left(\delta\left(\Delta_{1}\right), \ldots, \delta\left(\Delta_{k}\right)\right) .
$$

Using this bijection we get the Langlands classification in terms of $M\left(\mathscr{Y}_{F}\right)$.

For $a \in M\left(\mathscr{Y}_{F}\right)$ we set

$$
L(a)=L(M(\delta)(a)) \quad \text { and } \quad \lambda(a)=\lambda(M(\delta)(a)) .
$$

1.11. Note that $\S 1.10$ and the proposition in $\S 1.7$ imply that $R_{F}$ is a polynomial algebra over $\delta(\Delta), \Delta \in \mathscr{Y}_{F}$. Therefore the mapping

$$
\delta(\Delta) \mapsto \delta(\Delta)^{t}, \quad \Delta \in \mathscr{Y}_{F},
$$

extends uniquely to a ring morphism ${ }^{t}: R_{F} \rightarrow R_{F}$. This ring morphism is involutive. A fundamental fact is that it carries irreducible representations into irreducible ones; see [Aubert 1995; Schneider and Stuhler 1997].

Obviously, for a character $\chi$ of $F^{\times},(\chi \delta(\Delta))^{t} \cong \chi\left(\delta(\Delta)^{t}\right)$ for $\Delta \in \mathscr{Y}_{F}$. Therefore, ${ }^{t}: R_{F} \rightarrow R_{F}$ and $\chi: R_{F} \rightarrow R_{F}$ commute, since they commute on generators.

1.12. For an irreducible representation $\pi$ of a general linear group, there exists a unique $\left(\rho_{1}, \ldots, \rho_{k}\right) \in M\left(\mathscr{C}_{F}\right)$ such that

$$
\pi \hookrightarrow \rho_{1} \times \ldots \times \rho_{k} .
$$

The multiset $\left(\rho_{1}, \ldots, \rho_{k}\right)$ is called the cuspidal support of $\pi$ and it is denoted by

$$
\operatorname{supp}(\pi) \text {. }
$$

It is well-known (and one easily sees it) that ${ }^{t}: R_{F} \rightarrow R_{F}$ preserves the cuspidal support of irreducible representations.

1.13. Denote the set of all unitarizable classes in $\mathscr{D}_{F}$ by $\mathscr{D}_{F}^{u}$ (so $\mathscr{D}_{F}^{u}$ consists of the square-integrable classes). For $\delta \in D^{u}$ and a positive integer $n$ denote

$$
u(\delta, n)=L\left(v^{(n-1) / 2} \delta, v^{(n-3) / 2} \delta, \ldots, v^{-(n-1) / 2} \delta\right) .
$$


The following theorem describes irreducible unitarizable representations.

Theorem [Tadić 1986]. Let

$$
\mathscr{B}_{F}=\left\{u(\delta, n), \quad v^{\alpha} u(\delta, n) \times v^{-\alpha} u(\delta, n) \mid \delta \in \mathscr{D}_{F}^{u}, \quad n \in \mathbb{N}, \quad 0<\alpha<\frac{1}{2}\right\} .
$$

(i) If $\sigma_{1}, \ldots, \sigma_{k} \in \mathscr{B}_{F}$, then $\sigma_{1} \times \ldots \times \sigma_{k}$ is an irreducible unitarizable representation of some general linear group over $F$.

(ii) If $\pi$ is an irreducible unitarizable representation of some general linear group over $F$, there exist $\sigma_{1}, \ldots, \sigma_{m} \in \mathscr{B}_{F}$, unique up to a permutation, such that

$$
\pi \cong \sigma_{1} \times \ldots \times \sigma_{m} .
$$

1.14. Let $\rho \in \mathscr{C}_{F}$. Fix positive integers $d$ and $n$. Set

$$
\begin{aligned}
& \langle 1, d\rangle^{\rho}=\left[v^{-(d-1) / 2} \rho, v^{(d-1) / 2} \rho\right] \in \mathscr{Y}_{F}, \\
& \langle n, d\rangle^{\rho}=\left(\langle 1, d\rangle^{\nu^{-(n-1) / 2} \rho},\langle 1, d\rangle^{\nu^{1-(n-1) / 2} \rho}, \ldots,\langle 1, d\rangle^{\nu^{(n-1) / 2} \rho}\right) \in M\left(\mathscr{Y}_{F}\right) .
\end{aligned}
$$

We take (formally) $\langle 0, d\rangle^{\rho}$ to be the empty multiset (then $L\left(\langle 0, d\rangle^{\rho}\right.$ ) is the onedimensional representation of the trivial group $\operatorname{GL}(0, F)$, which is the identity of $R_{F}$ ). Similarly, we also take $\langle n, 0\rangle^{\rho}$ to be the empty multiset (so again $L\left(\langle n, 0\rangle^{\rho}\right.$ ) is the identity in $R$ ). Observe that

$$
\begin{aligned}
{\left[v^{k_{1}} \rho, v^{k_{2}} \rho\right] } & =\left[k_{1}, k_{2}\right]^{\rho}=\left\langle 1, k_{2}-k_{1}+1\right\rangle^{v^{\left(k_{1}+k_{2}\right) / 2} \rho}, \\
\langle 1, d\rangle^{v^{\alpha} \rho} & =[-(d-1) / 2+\alpha,(d-1) / 2+\alpha]^{\rho} .
\end{aligned}
$$

From $\S 1.7$ follows that for a character $\chi$ of $F^{\times}$

$$
\chi L\left(\langle n, d\rangle^{\rho}\right) \cong L\left(\langle n, d\rangle^{\chi \rho}\right) .
$$

Further

$$
u(\delta(\rho, d), n)=L\left(\langle n, d\rangle^{\rho}\right) .
$$

1.15. There are two important distinguished bases of $R_{F}$, irreducible representations and standard modules $\lambda(d), d \in M\left(\mathscr{D}_{F}\right)$. The theorem in $\S 1.13$ implies that the following theorem solves the problem of expressing irreducible unitary representations in terms of standard modules, and irreducible unitary characters in terms of standard characters. It is convenient to present it in the following form:

Theorem [Tadić 1995]. Let $n, d \in \mathbb{Z}, n, d \geq 1$. Let $W_{n}$ be the group of permutations of the set $\{1,2, \ldots, n\}$. Set $W_{n}^{(d)}=\left\{w \in W_{n} ; w(i)+d \geq i\right.$ for all $\left.1 \leq i \leq n\right\}$. Then

$$
L\left(\left(\left[v \rho, v^{d} \rho\right], \ldots,\left[v^{n} \rho, v^{d+n-1} \rho\right]\right)\right)=\sum_{w \in W_{n}^{(d)}}(-1)^{w} \prod_{i=1}^{n} \delta\left(\left[v^{i} \rho, v^{w(i)+(d-1)} \rho\right]\right)
$$

in $R_{F}$, where $(-1)^{w}$ denotes the sign of the permutation $w$. 
1.16. From the following theorem one can get all the irreducible subquotients of ends of complementary series. (This is crucial information for determining the topology of the unitary dual.)

Theorem [Tadić 1987]. For positive integers $n$ and $d$, and $\rho \in \mathscr{C}_{F}$, we have

$$
\begin{aligned}
& v^{1 / 2} L\left(\langle n, d\rangle^{\rho}\right) \times v^{-1 / 2} L\left(\langle n, d\rangle^{\rho}\right) \\
& \quad=L\left(\langle n+1, d\rangle^{\rho}\right) \times L\left(\langle n-1, d\rangle^{\rho}\right)+L\left(\langle n, d+1\rangle^{\rho}\right) \times L\left(\langle n, d-1\rangle^{\rho}\right) .
\end{aligned}
$$

1.17. The following theorem implies that the involution ${ }^{t}$ carries class of irreducible unitary representations to itself. Moreover, it implies an explicit formula for the involution on irreducible unitary representations.

Theorem [Tadić 1986]. For positive integers $n$ and $d$, and $\rho \in \mathscr{C}_{F}$, we have

$$
\left(L\left(\langle n, d\rangle^{\rho}\right)\right)^{t}=L\left(\langle d, n\rangle^{\rho}\right) .
$$

\section{Representations of GL( $(n)$ over a division algebra $A$}

We now introduce necessary notation and basic results on general linear groups over division algebras. Since the situation is very similar to the case of general linear groups over a field, we only point out the differences between these two cases. (More details can be found in [Tadić 1990].)

We assume that the characteristic of $F$ is 0 .

2.1. Let $A$ be a finite dimensional division algebra over $F$ whose center is $F$. Let

$$
\operatorname{dim}_{F} A=d_{A}^{2} .
$$

Let Mat $(n \times n, A)$ be the algebra of all $n \times n$ matrices with entries in $A$. Then $\operatorname{GL}(n, A)$ is the group of invertible matrices with the natural topology. The commutator subgroup is denoted by $\operatorname{SL}(n, A)$. We denote by

$$
\operatorname{det}: \mathrm{GL}(n, A) \rightarrow \mathrm{GL}(1, A) / \mathrm{SL}(1, A)
$$

the determinant homomorphism, as defined by J. Dieudonné (for $n=1$ this is just the quotient map). The kernel is $\operatorname{SL}(n, A)$.

The reduced norm of $\operatorname{Mat}(n \times n, A)$ will be denoted by RN. We identify characters of $\operatorname{GL}(n, A)$ with characters of $F^{\times}$using RN. Let

$$
v=|\mathrm{RN}|_{F}: \operatorname{GL}(n, A) \rightarrow \mathbb{R}^{\times} .
$$

2.2. We now comment on modifications that need to be made to the material in Sections 1.3 to 1.12 so it applies also to the case of general linear groups over division algebras. (More details can be found in [Tadić 1990].) Only small modifications are required for $§ 1.3-\$ 1.8$; we deal with them first. 
1.3: We define by the same formula as in $\S 1.3$ the multiplication $\times$ of smooth representations of general linear groups over $A$. Again as in $\S 1.3$, we have $\left(\pi_{1} \times \pi_{2}\right) \times \pi_{3} \cong \pi_{1} \times\left(\pi_{2} \times \pi_{3}\right)$.

1.4: In $\$ 2.1$ we identified characters of $\operatorname{GL}(n, A)$ with characters of $F^{\times}$using the reduced norm RN. The character identified with ||$_{F}$ was again denoted by $v$. Again, for a character $\chi$ of $F^{\times}$, we have $\chi\left(\pi_{1} \times \pi_{2}\right) \cong\left(\chi \pi_{1}\right) \times\left(\chi \pi_{2}\right)$.

1.5: Define

$$
R_{n, A}=\operatorname{Groth} \mathrm{GL}(n, A)
$$

(recall that $R_{F}=\bigoplus_{n \geq 0} R_{n, F}$ ). One defines on $R_{A}$ the structure of an (associative, commutative) ring in the same way as in $\S 1.5$ was done for the field case. Also, characters of $F^{\times}$lift to automorphisms of $R_{A}$ (as in $\S 1.5$ ).

1.6: All essentially square-integrable classes in $\bigcup_{n \geq 1} \operatorname{GL}(n, A)^{\sim}$ are denoted by $\mathscr{D}_{A}$. One defines $e(\delta)$ and $\delta^{u}$ for $\delta \in \mathscr{D}_{A}$ as in $\S 1.6$.

1.7: For $d \in M\left(\mathscr{D}_{A}\right)$ we define $\lambda(d)$ and $L(d)$ in the same way as in $\S 1.7$. The Langlands classification for general linear groups over division algebras have the same expression as in the field case. (The parameters are in $M\left(\mathscr{D}_{A}\right)$.)

Here $R_{A}$ is a polynomial algebra over $\mathscr{D}_{A}$.

1.8: The proposition holds in the same form for general linear groups over division algebras; see [Tadić 1990, Proposition 2.3].

2.3. By [Deligne et al. 1984] there exists a bijection

$$
\mathrm{JL}_{p}: \mathscr{D}(\mathrm{GL}(p, A)) \rightarrow \mathscr{D}\left(\mathrm{GL}\left(p d_{A}, F\right)\right), \quad \delta^{\prime} \leftrightarrow \delta,
$$

such that characters $\Theta_{\delta^{\prime}}$ and $\Theta_{\delta}$ satisfy

$$
(-1)^{p} \Theta_{\delta^{\prime}}\left(g^{\prime}\right)=(-1)^{p d_{A}} \Theta_{\delta}(g)
$$

whenever $g^{\prime}$ and $g$ have same characteristic polynomials, and when this polynomial is separable.

This bijection is uniquely determined by the character requirement above and is called the Jacquet-Langlands correspondence between irreducible essentially square-integrable representations of $\operatorname{GL}(p, A)$ and $\operatorname{GL}\left(p d_{A}, F\right)$.

This bijection commutes with twisting with characters (see [Badulescu 2002]).

Take $\delta^{\prime} \in \mathscr{D}(\mathrm{GL}(p, A))$ cuspidal and let $\delta^{\prime}$ correspond to $\delta \in \mathscr{D}\left(\mathrm{GL}\left(p d_{A}, F\right)\right)$ by the correspondence above. We know that

$$
\delta=\delta(\rho, q)
$$

for some positive integer $q$ which divides $p d_{A}$ and for some irreducible cuspidal representation $\rho$ of $\operatorname{GL}\left(\left(p d_{A}\right) / q, F\right)$. Further, it is known that $q \mid d_{A}$ and that $p$ is relatively prime to $q$ ( $p d_{A}$ is the lowest common multiple of $d_{A}$ and $\left(p d_{A}\right) / q$ ). 
We define

$$
s_{\delta^{\prime}}=q,
$$

and a character

$$
v_{\delta^{\prime}}=v^{s_{\delta^{\prime}}}
$$

of $F^{\times}$. As we noted in $\S 2.1$, we use RN to identify the characters of $F^{\times}$with characters of $\operatorname{GL}(n, A)$. Therefore, we can view $v_{\delta^{\prime}}$ as a character of any $\operatorname{GL}(k, A)$. (Note that in this definition, $\delta^{\prime}$ is cuspidal; soon we shall give a definition also in the case that $\delta^{\prime}$ is essentially square-integrable.)

2.4. We continue with the modifications required so the material in $\S 1.9$ to $\S 1.12$ can apply to general linear groups over division algebras.

1.9: We denote by $\mathscr{C}_{A}$ the set of equivalence classes of irreducible cuspidal representation of all $\operatorname{GL}(n, A), n \geq 1$. For $\rho^{\prime} \in \mathscr{C}_{A}$ and $k \in \mathbb{Z}, k \geq 0$, the set

$$
\left[\rho^{\prime}, v_{\rho^{\prime}}^{k} \rho^{\prime}\right]=\left\{\rho^{\prime}, v_{\rho^{\prime}} \rho^{\prime}, v_{\rho^{\prime}}^{2} \rho^{\prime}, \ldots, v_{\rho^{\prime}}^{k} \rho^{\prime}\right\}
$$

is called a segment of irreducible cuspidal representations of general linear groups over division algebras. In this case, we also denote the segment $\left[v_{\rho^{\prime}}^{k_{1}} \rho^{\prime}, v_{\rho^{\prime}}^{k_{2}} \rho^{\prime}\right]$ (for $k_{1}, k_{2} \in \mathbb{R}, k_{2}-k_{1} \in \mathbb{Z}$ and $k_{2}-k_{1} \geq 0$ ) by

$$
\left[k_{1}, k_{2}\right]^{\rho^{\prime}} .
$$

The set of all such segments will be denoted by $\mathscr{S}_{A}$. The set of all finite multisets in $\mathscr{S}_{A}$ will be denoted by $M\left(\mathscr{Y}_{F}\right)$ and we consider the partial ordering $\leq$ on $M\left(\mathscr{S}_{A}\right)$ introduced in [Tadić 1990 , Section 4] (defined again by linking segments).

1.10: For $\Delta^{\prime}=\left[\rho^{\prime}, v_{\rho^{\prime}}^{k} \rho^{\prime}\right]=\left\{\rho^{\prime}, v_{\rho^{\prime}} \rho^{\prime}, v_{\rho^{\prime}}^{2} \rho^{\prime}, \ldots, v_{\rho^{\prime}}^{k} \rho^{\prime}\right\} \in \mathscr{Y}_{A}$, the representation $\rho^{\prime} \times v_{\rho^{\prime}} \rho^{\prime} \times v_{\rho^{\prime}}^{2} \rho^{\prime} \times \ldots \times v_{\rho^{\prime}}^{k} \rho^{\prime}$ has a unique irreducible quotient, denoted by $\delta\left(\left[\rho^{\prime}, v_{\rho^{\prime}}^{k} \rho^{\prime}\right]\right)$, and it has a unique irreducible subrepresentation, denoted by $\delta\left(\left[\rho^{\prime}, v_{\rho^{\prime}}^{k} \rho^{\prime}\right]\right)^{t}$.

The mapping

$$
\delta: \mathscr{Y}_{A} \rightarrow \mathscr{D}_{A}, \quad \Delta^{\prime} \mapsto \delta\left(\Delta^{\prime}\right),
$$

is a bijection. If we let

$$
\delta\left(\rho^{\prime}, n\right)=\delta\left([-(n-1) / 2,(n-1) / 2]^{\rho^{\prime}}\right),
$$

then we can restate this as follows: $\left(\rho^{\prime}, n\right) \mapsto \delta\left(\rho^{\prime}, n\right)$ is a bijection of $\mathscr{C}_{A} \times \mathbb{N}$ onto $\mathscr{D}_{A}$.

We define $v_{\delta\left(\rho^{\prime}, n\right)}$ to be $v_{\rho^{\prime}}$, i.e.

$$
v_{\delta\left(\rho^{\prime}, n\right)}=v_{\rho^{\prime}} .
$$


We lift $\Delta^{\prime} \mapsto \delta\left(\Delta^{\prime}\right)$ naturally to a bijection $M(\delta): M\left(\mathscr{Y}_{A}\right) \rightarrow M\left(\mathscr{D}_{A}\right)$. This gives the Langlands classification for general linear groups over a division algebra $A$ in terms of $M\left(\mathscr{Y}_{A}\right)$. For $a \in M\left(\mathscr{Y}_{A}\right)$ let $L(a)=L(M(\delta)(a))$ and $\lambda(a)=\lambda(M(\delta)(a))$ as before.

1.11: Since $R_{A}$ is a polynomial algebra over $\delta\left(\Delta^{\prime}\right), \Delta^{\prime} \in \mathscr{Y}_{A}$, the mapping $\delta\left(\Delta^{\prime}\right) \mapsto$ $\delta\left(\Delta^{\prime}\right)^{t}, \quad \Delta \in \mathscr{Y}_{A}$, extends uniquely to the ring morphism ${ }^{t}: R_{A} \rightarrow R_{A}$, which carries irreducible representations into irreducible ones; see [Aubert 1995; Schneider and Stuhler 1997]. This homomorphism of rings is an involution. Again, for a character $\chi$ of $F^{\times},{ }^{t}: R_{A} \rightarrow R_{A}$ and $\chi: R_{A} \rightarrow R_{A}$ commute.

1.12: One defines the cuspidal support of an irreducible representation in the same way as before (it is an element of $\left.M\left(\mathscr{C}_{A}\right)\right)$. The involution ${ }^{t}$ preserves the cuspidal support.

2.5. Let $\mathscr{D}_{A}^{u}$ be the set of all the unitarizable classes in $\mathscr{D}_{A}$. Let

$$
u\left(\delta^{\prime}, n\right)=L\left(v_{\delta^{\prime}}^{(n-1) / 2} \delta^{\prime}, v_{\delta^{\prime}}^{(n-3) / 2} \delta^{\prime}, \ldots, v_{\delta^{\prime}}^{-(n-1) / 2} \delta^{\prime}\right) .
$$

for $\delta^{\prime} \in D_{A}^{u}$ and a positive integer $n$.

We first recall a conjecture from [Tadić 1990]:

$$
\text { If } \pi_{1} \text { and } \pi_{2} \text { are irreducible unitarizable representations of }
$$
general linear groups over $A$, then $\pi_{1} \times \pi_{2}$ is irreducible.

Now [Tadić 1990, Section 6] and [Badulescu and Renard 2004] imply:

Proposition. Assume that (U0) holds. Let

$$
\mathscr{B}_{A}=\left\{u\left(\delta^{\prime}, n\right), \quad v_{\delta^{\prime}}^{\alpha} u\left(\delta^{\prime}, n\right) \times v_{\delta^{\prime}}^{-\alpha} u\left(\delta^{\prime}, n\right) \mid \delta^{\prime} \in \mathscr{D}_{A}^{u}, \quad n \in \mathbb{N}, \quad 0<\alpha<\frac{1}{2}\right\} .
$$

Then

(i) If $\sigma_{1}, \ldots, \sigma_{k} \in \mathscr{B}_{A}$, then $\sigma_{1} \times \ldots \times \sigma_{k}$ is an irreducible unitarizable representation of some general linear group over $A$.

(ii) If $\pi$ is an irreducible unitarizable representation of some general linear group over $A$, then there exist $\sigma_{1}, \ldots, \sigma_{m} \in \mathscr{B}_{A}$, unique up to a permutation, such that

$$
\pi \cong \sigma_{1} \times \ldots \times \sigma_{m}
$$

2.6. For $\rho^{\prime} \in \mathscr{C}_{A}$ and positive integers $d, n$, set

$$
\begin{aligned}
& \langle 1, d\rangle^{\rho^{\prime}}=\left[v_{\rho^{\prime}}^{-(d-1) / 2} \rho^{\prime}, v_{\rho^{\prime}}^{(d-1) / 2} \rho^{\prime}\right] \in \mathscr{T}_{A}, \\
& \langle n, d\rangle^{\rho^{\prime}}=\left(\langle 1, d\rangle^{v_{\rho^{\prime}}^{-(n-1) / 2} \rho^{\prime}},\langle 1, d\rangle^{v_{\rho^{\prime}}^{1-(n-1) / 2} \rho^{\prime}}, \ldots,\langle 1, d\rangle^{v_{\rho^{\prime}}^{(n-1) / 2} \rho^{\prime}}\right) \in M\left(\mathscr{S}_{A}\right) .
\end{aligned}
$$


Further, $\langle 0, d\rangle^{\rho^{\prime}}$ and $\langle n, 0\rangle^{\rho^{\prime}}$ are empty multisets (so $L\left(\langle 0, d\rangle^{\rho^{\prime}}\right.$ ) and $L\left(\langle n, 0\rangle^{\rho^{\prime}}\right.$ ) are both the identity in $R_{A}$ ). As before, we have

$$
\begin{aligned}
{\left[v_{\rho^{\prime}}^{k_{1}} \rho^{\prime}, v_{\rho^{\prime}}^{k_{2}} \rho^{\prime}\right] } & =\left[k_{1}, k_{2}\right]^{\rho^{\prime}}=\left\langle 1, k_{2}-k_{1}+1\right\rangle^{v_{\rho^{\prime}}^{\left(k_{1}+k_{2}\right) / 2} \rho^{\prime}}, \\
\langle 1, d\rangle^{\nu_{\rho^{\prime}}^{\alpha} \rho^{\prime}} & =[-(d-1) / 2+\alpha,(d-1) / 2+\alpha]^{\rho^{\prime}} .
\end{aligned}
$$

For a character $\chi$ of $F^{\times}$we have $\chi L\left(\langle n, d\rangle^{\rho^{\prime}}\right) \cong L\left(\langle n, d\rangle^{\chi \rho^{\prime}}\right)$. Also

$$
u\left(\delta\left(\rho^{\prime}, d\right), n\right)=L\left(\langle n, d\rangle^{\rho^{\prime}}\right) .
$$

\section{Involution and unitarity on unitary duals of $\operatorname{GL}(n, A)$}

3.1. We call an irreducible representation $\pi$ of a general linear group over $A$ rigid if

$$
e\left(\rho^{\prime}\right) / s_{\rho^{\prime}} \in \frac{1}{2} \mathbb{Z}
$$

for all $\rho^{\prime}$ in the cuspidal support of $\pi$.

Lemma. Assume that (U0) holds. For $d, n \in \mathbb{N}, \rho^{\prime} \in \mathscr{C}_{A}$ we have

$$
L\left(\langle n, d\rangle^{\rho^{\prime}}\right)^{t}=L\left(\langle d, n\rangle^{\rho^{\prime}}\right) .
$$

Proof. Since ${ }^{t}$ commutes with character automorphisms $\chi: R_{A} \rightarrow R_{A}$, it is enough to prove the equality for unitary $\rho^{\prime} \in \mathscr{C}_{A}$.

The proof goes in several steps.

First we prove that $L\left(\langle n, d\rangle^{\rho^{\prime}}\right)^{t}$ is unitarizable. We prove it by induction with respect to $n$. For $n=1$ we know $L\left(\langle 1, d\rangle^{\rho^{\prime}}\right)^{t}=L\left(\langle d, 1\rangle^{\rho^{\prime}}\right)$, which is unitarizable by the proposition in $\S 2.5$.

Let $n \geq 1$ and suppose that we have proved the unitarity of the representations $L\left(\langle n, d\rangle^{\rho^{\prime}}\right)^{t}$ for that $n$. The division algebra version of the proposition from $\S 1.8$ (see §2.2), together with (U0), implies that

$$
L\left(\langle n+1, d\rangle^{\rho^{\prime}}\right) \times L\left(\langle n-1, d\rangle^{\rho^{\prime}}\right) \leq v_{\rho^{\prime}}^{-1 / 2} L\left(\langle n, d\rangle^{\rho^{\prime}}\right) \times v_{\rho^{\prime}}^{1 / 2} L\left(\langle n, d\rangle^{\rho^{\prime}}\right) .
$$

Applying the involution ${ }^{t}: R_{A} \rightarrow R_{A}$ to this relation, we get

$$
L\left(\langle n+1, d\rangle^{\rho^{\prime}}\right)^{t} \times L\left(\langle n-1, d\rangle^{\rho^{\prime}}\right)^{t} \leq v_{\rho^{\prime}}^{-1 / 2} L\left(\langle n, d\rangle^{\rho^{\prime}}\right)^{t} \times v_{\rho^{\prime}}^{1 / 2} L\left(\langle n, d\rangle^{\rho^{\prime}}\right)^{t} .
$$

First observe that $L\left(\langle n, d\rangle^{\rho^{\prime}}\right)^{t}$ is rigid (since ${ }^{t}$ preserves the cuspidal support). This fact, the unitarity of $L\left(\langle n, d\rangle^{\rho^{\prime}}\right)^{t}$ and The proposition in $\$ 2.5$ imply that $L\left(\langle n, d\rangle^{\rho^{\prime}}\right)^{t}$ is a product of elements of the form $L\left(\left\langle n^{\prime}, d^{\prime}\right\rangle^{\rho^{\prime \prime}}\right)$, where $\rho^{\prime \prime} \in \mathscr{C}_{A}$ are unitarizable. The same proposition implies that all representations

$$
v_{\rho^{\prime \prime}}^{-\alpha} L\left(\left\langle n^{\prime}, d^{\prime}\right\rangle^{\rho^{\prime \prime}}\right) \times v_{\rho^{\prime \prime}}^{\alpha} L\left(\left\langle n^{\prime}, d^{\prime}\right\rangle^{\rho^{\prime \prime}}\right), \quad 0<\alpha<\frac{1}{2},
$$


are unitarizable. This implies that the representations

$$
v_{\rho^{\prime}}^{-\alpha} L\left(\langle n, d\rangle^{\rho^{\prime}}\right)^{t} \times v_{\rho^{\prime}}^{\alpha} L\left(\langle n, d\rangle^{\rho^{\prime}}\right)^{t}, \quad 0<\alpha<\frac{1}{2},
$$

are also unitarizable. Recall that all the irreducible subquotients at the end of complementary series are unitarizable ([Miličić 1973], see also [Tadić 1986] and [Tadić 1988]). This implies that all the irreducible subquotients of $v_{\rho^{\prime}}^{-1 / 2} L\left(\langle n, d\rangle^{\rho^{\prime}}\right)^{t} \times$ $v_{\rho^{\prime}}^{1 / 2} L\left(\langle n, d\rangle^{\rho^{\prime}}\right)^{t}$ are unitarizable. In particular,

$$
L\left(\langle n+1, d\rangle^{\rho^{\prime}}\right)^{t} \times L\left(\langle n-1, d\rangle^{\rho^{\prime}}\right)^{t}
$$

is unitarizable (and irreducible, since ${ }^{t}$ carries irreducible representations to the irreducible ones).

For an irreducible representation $\pi$ denote by $\pi^{+}$the Hermitian contragredient of $\pi$. Then $\pi \mapsto \pi^{+}$lifts to an automorphism of $R_{A}$. Observe that ${ }^{t}$ carries the class of irreducible Hermitian representations to itself, since the automorphisms ${ }^{t}$ and $\pi \mapsto \pi^{+}$of $R_{A}$ commute (one directly checks this on generators). Therefore, $L\left(\langle n+1, d\rangle^{\rho^{\prime}}\right)^{t} \otimes L\left(\langle n-1, d\rangle^{\rho^{\prime}}\right)^{t}$ is Hermitian. Now (d) in section 3 of [Tadić 1993] implies that $L\left(\langle n+1, d\rangle^{\rho^{\prime}}\right)^{t} \otimes L\left(\langle n-1, d\rangle^{\rho^{\prime}}\right)^{t}$ is (irreducible) unitarizable, which implies that $L\left(\langle n+1, d\rangle^{\rho^{\prime}}\right)^{t}$ is unitarizable. Therefore, we have proved the inductive step.

So, we have proved that representations $L\left(\langle n, d\rangle^{\rho^{\prime}}\right)^{t}$ for $\rho^{\prime} \in \mathscr{C}_{A}$ unitarizable, are unitarizable in general.

We will now get an explicit formula for $L\left(\langle n, d\rangle^{\rho^{\prime}}\right)^{t}$.

First note that $L\left(\langle n, d\rangle^{\rho^{\prime}}\right)$ is not induced from a proper parabolic subgroup by an irreducible unitarizable representation (see Proposition 2.5). Therefore, $L\left(\langle n, d\rangle^{\rho^{\prime}}\right)^{t}$ is also not induced in that way. The proposition in $\$ 2.5$ implies that $\left(L\left(\langle n, d\rangle^{\rho^{\prime}}\right)\right)^{t}=L\left(\left\langle n^{\prime}, d^{\prime}\right\rangle^{\rho^{\prime \prime}}\right)$ for some $n^{\prime}, d^{\prime}$ and $\rho^{\prime \prime}$. Since ${ }^{t}$ preserves the cuspidal support, one gets directly $\rho^{\prime} \cong \rho^{\prime \prime}$ and $\{n, d\}=\left\{n^{\prime}, d^{\prime}\right\}$. This implies the lemma if $n=d$.

It remains to consider the case $n \neq d$. Actually, in this case it is enough to prove

$$
L\left(\langle n, d\rangle^{\rho^{\prime}}\right)^{t} \neq L\left(\langle n, d\rangle^{\rho^{\prime}}\right) .
$$

Since ${ }^{t}$ is an involution, our previous discussion implies that it is enough to prove this relation in the case

$$
d<n .
$$

We do this by induction on $d$. For $d=1$,

$$
L\left(\langle n, 1\rangle^{\rho^{\prime}}\right)^{t}=L\left(\langle 1, n\rangle^{\rho^{\prime}}\right),
$$

which is different from $L\left(\langle n, 1\rangle^{\rho^{\prime}}\right)$ since $n>1$. 
Suppose $d \geq 1$ and that we have proved the claim for $d^{\prime} \leq d$. Let $d+1<n$. We have already observed that $L\left(\langle n+1, d\rangle^{\rho^{\prime}}\right) \times L\left(\langle n-1, d\rangle^{\rho^{\prime}}\right) \leq v_{\rho}^{-1 / 2} L\left(\langle n, d\rangle^{\rho^{\prime}}\right) \times$ $v_{\rho}^{1 / 2} L\left(\langle n, d\rangle^{\rho^{\prime}}\right)$. Applying ${ }^{t}$ to this relation and using the inductive assumption, we get

$$
L\left(\langle n+1, d\rangle^{\rho^{\prime}}\right)^{t} \times L\left(\langle d, n-1\rangle^{\rho^{\prime}}\right) \leq v_{\rho}^{-1 / 2} L\left(\langle d, n\rangle^{\rho^{\prime}}\right) \times v_{\rho}^{1 / 2} L\left(\langle d, n\rangle^{\rho^{\prime}}\right) .
$$

Suppose

$$
L\left(\langle n+1, d\rangle^{\rho^{\prime}}\right)^{t}=L\left(\langle n+1, d\rangle^{\rho^{\prime}}\right) .
$$

Then

$$
L\left(\langle n+1, d\rangle^{\rho^{\prime}}\right) \times L\left(\langle d, n-1\rangle^{\rho^{\prime}}\right) \leq v_{\rho}^{-1 / 2} L\left(\langle d, n\rangle^{\rho^{\prime}}\right) \times v_{\rho}^{1 / 2} L\left(\langle d, n\rangle^{\rho^{\prime}}\right) .
$$

Then by the definition of the ordering $\leq$ on $M\left(\mathscr{Y}_{A}\right)$, we can not have on the left hand side more segments than on the right hand side (since ordering is generated by linking segments). This implies $n+1+d \leq 2 d$, i.e., $n+1 \leq d$ which implies $n<d$. This contradicts $d+1<n$. Thus $L\left(\langle n+1, d\rangle^{\rho^{\prime}}\right)^{t} \neq L\left(\langle n+1, d\rangle^{\rho^{\prime}}\right)$, what we needed to prove.

3.2. Corollary. Assume that (U0) holds. Then ${ }^{t}$ carries the class of irreducible unitary representations to itself.

\section{On ends of complementary series of $\operatorname{GL}(n, A)$; character identities}

4.1. The following proposition describes irreducible subquotients in the ends of complementary series. Besides the fact that this is crucial information for determining the topology of the unitary dual, this result (essentially character identity) will be crucial for us in obtaining formulas for (characters of) irreducible unitary representations in terms of (characters of) standard modules.

Proposition. Assume that (U0) holds. Then for $n, d \in \mathbb{N}, \rho^{\prime} \in \mathscr{C}_{A}$ we have in $R_{A}$

$$
\begin{aligned}
& v_{\rho^{\prime}}^{-1 / 2} L\left(\langle n, d\rangle^{\rho^{\prime}}\right) \times v_{\rho^{\prime}}^{1 / 2} L\left(\langle n, d\rangle^{\rho^{\prime}}\right)= \\
& L\left(\langle n-1, d\rangle^{\rho^{\prime}}\right) \times L\left(\langle n+1, d\rangle^{\rho^{\prime}}\right)+L\left(\langle n, d-1\rangle^{\rho^{\prime}}\right) \times L\left(\langle n, d+1\rangle^{\rho^{\prime}}\right) .
\end{aligned}
$$

Proof. It is enough to prove the equality for $\rho^{\prime}$ unitarizable. Further, by [Tadić 1990, Proposition 4.3], it is enough to prove the proposition for $n \geq 2$. Applying involution ${ }^{t}$, we conclude that it is enough to consider only the case $d \geq 2$.

Let

$$
\pi=v_{\rho^{\prime}}^{-1 / 2} L\left(\langle n, d\rangle^{\rho^{\prime}}\right) \times v_{\rho^{\prime}}^{1 / 2} L\left(\langle n, d\rangle^{\rho^{\prime}}\right) .
$$

We know that $L\left(\langle n-1, d\rangle^{\rho^{\prime}}\right) \times L\left(\langle n+1, d\rangle^{\rho^{\prime}}\right)$ is a subquotient of multiplicity one in $\pi$ (see $\$ 2.2)$. 
Applying the same argument to $\pi^{\prime}=v_{\rho^{\prime}}^{-1 / 2} L\left(\langle d, n\rangle^{\rho^{\prime}}\right) \times v_{\rho^{\prime}}^{1 / 2} L\left(\langle d, n\rangle^{\rho^{\prime}}\right)$, we get that $L\left(\langle d-1, n\rangle^{\rho^{\prime}}\right) \times L\left(\langle d+1, n\rangle^{\rho^{\prime}}\right)$ is a subquotient of $\pi^{\prime}$ of multiplicity one.

Applying ${ }^{t}$ to $\pi^{\prime}$, we get from the lemma in $\S 3.1$ that $L\left(\langle n, d-1\rangle^{\rho^{\prime}}\right) \times L(\langle n, d+$ $\left.1\rangle^{\rho^{\prime}}\right)$ is a subquotient of $\pi=\left(\pi^{\prime}\right)^{t}$ of multiplicity one. Therefore, to complete the proof, it is enough to prove that there are no additional irreducible subquotients besides these two.

Let $\sigma$ be an irreducible subquotient of $\pi$ different from the two subquotients above. Since $\pi$ is an end of complementary series, $\sigma$ must be unitarizable. Since $\pi$ is rigid, $\sigma$ must be rigid. This easily implies that

$$
\sigma=L\left(\left\langle n_{1}, d_{1}\right\rangle^{\rho^{\prime}}\right) \times \ldots \times L\left(\left\langle n_{k}, d_{k}\right\rangle^{\rho^{\prime}}\right)
$$

for some $n_{i}$ 's and $d_{i}$ 's. After renumbering, we can (and shall) assume that

$$
n_{1}+d_{1} \geq n_{2}+d_{2} \geq \cdots \geq n_{k}+d_{k} .
$$

Look at the cuspidal representation $v_{\rho^{\prime}}^{-(n+d) / 2+1-1 / 2} \rho^{\prime}=v_{\rho^{\prime}}^{-(n+d) / 2+1 / 2} \rho^{\prime}$. This is the first representation (from the negative left hand side) in the cuspidal support of $\pi$. Then the cuspidal support tells

$$
n_{1}+d_{1}=n+d+1
$$

(We must have $n_{1}+d_{1}>n_{2}+d_{2}$, since the multiplicity of $\nu_{\rho^{\prime}}^{-(n+d) / 2+1 / 2} \rho^{\prime}$ in the cuspidal support of $\pi$ is one.)

The rules for linking segments imply

$$
d \leq d_{1} .
$$

(Since $v_{\rho^{\prime}}^{-(n+d) / 2+1 / 2} \rho^{\prime}$ is the left end of only one segment in $\pi$, and there are no segments which are more to the left, the segment starting with $v_{\rho^{\prime}}^{-(n+d) / 2+1 / 2} \rho^{\prime}$ must be at least of length $d$.) Applying ${ }^{t}$, invoking the lemma in $\S 3.1$, and repeating the preceding argument, we get

$$
n \leq n_{1}
$$

The three relations above imply

$$
\left(n_{1}, d_{1}\right)=(n+1, d) \quad \text { or } \quad\left(n_{1}, d_{1}\right)=(n, d+1),
$$

that is,

$$
L\left(\left\langle n_{1}, d_{1}\right\rangle^{\rho^{\prime}}\right)=L\left(\langle n+1, d\rangle^{\rho^{\prime}}\right) \quad \text { or } \quad L\left(\left\langle n_{1}, d_{1}\right\rangle^{\rho^{\prime}}\right)=L\left(\langle n, d+1\rangle^{\rho^{\prime}}\right) .
$$

Now the first remaining representation in the cuspidal support is

$$
v_{\rho^{\prime}}^{-(n+d) / 2+1 / 2+1} \rho=v_{\rho^{\prime}}^{-(n+d) / 2+3 / 2} \rho .
$$


(As above, looking at the cuspidal support of $\pi$, we can conclude that $n_{2}+d_{2}>$ $n_{3}+d_{3}$ if $k \geq 3$.) This implies

$$
n_{2}+d_{2}=n+d-1
$$

Now looking at the rules for linking segments, one gets directly

$$
d-1 \leq d_{2}
$$

(Note that $v_{\rho^{\prime}}^{-(n+d) / 2+3 / 2} \rho^{\prime}$ must be the beginning of a segment in $\left\langle n_{2}, d_{2}\right\rangle^{\rho^{\prime}}$, and the shortest segment that can have this beginning is of length $d-1$, which one gets by intersecting the most left segment in $\pi$ with the segment in $\pi$ starting at $\left.v_{\rho^{\prime}}^{-(n+d) / 2+1 / 2} \rho^{\prime}\right)$.

Repeating the preceding argument in the case of $\pi^{t}$ and using the lemma in $\S 3.1$ we get

$$
n-1 \leq n_{2}
$$

The three relations above imply

$$
\left(n_{2}, d_{2}\right)=(n-1, d) \quad \text { or } \quad\left(n_{2}, d_{2}\right)=(n, d-1),
$$

that is,

$$
L\left(\left\langle n_{2}, d_{2}\right\rangle^{\rho^{\prime}}\right)=L\left(\langle n-1, d\rangle^{\rho^{\prime}}\right) \quad \text { or } \quad L\left(\left\langle n_{2}, d_{2}\right\rangle^{\rho^{\prime}}\right)=L\left(\langle n, d-1\rangle^{\rho^{\prime}}\right) .
$$

We now have four possibilities for the first two factors of $\sigma$. We shall analyze two possibilities. Let

$$
\sigma^{\prime}= \begin{cases}L\left(\left\langle n_{3}, d_{3}\right\rangle^{\rho^{\prime}}\right) \times \cdots \times L\left(\left\langle n_{k}, d_{k}\right\rangle^{\rho^{\prime}}\right) & \text { if } k \geq 3, \\ 1 & \text { otherwise. }\end{cases}
$$

Suppose that $\sigma$ is isomorphic to

$L\left(\langle n+1, d\rangle^{\rho^{\prime}}\right) \times L\left(\langle n, d-1\rangle^{\rho^{\prime}}\right) \times \sigma^{\prime} \quad$ or $\quad L\left(\langle n, d+1\rangle^{\rho^{\prime}}\right) \times L\left(\langle n-1, d\rangle^{\rho^{\prime}}\right) \times \sigma^{\prime}$.

The first representation cannot be a subquotient of $\pi$ since it corresponds to at least $2 n+1$ segments, while $\pi$ is defined by $2 n$ segments. For the second representation, observe that

$$
\left(L\left(\langle n, d+1\rangle^{\rho^{\prime}}\right) \times L\left(\langle n-1, d\rangle^{\rho^{\prime}}\right) \times \sigma\right)^{t}=L\left(\langle d+1, n\rangle^{\rho^{\prime}}\right) \times L\left(\langle d, n-1\rangle^{\rho^{\prime}}\right) \times\left(\sigma^{\prime}\right)^{t}
$$

is a subquotient of $\pi^{t}=v_{\rho^{\prime}}^{-1 / 2} L\left(\langle d, n\rangle^{\rho^{\prime}}\right) \times v_{\rho^{\prime}}^{1 / 2} L\left(\langle d, n\rangle^{\rho^{\prime}}\right)$. This is impossible, for the same reason as in the first case.

Therefore, the only two remaining possibilities for $\sigma$ are $L\left(\langle n-1, d\rangle^{\rho^{\prime}}\right) \times L\left(\langle n+1, d\rangle^{\rho^{\prime}}\right) \times \sigma^{\prime} \quad$ and $\quad L\left(\langle n, d-1\rangle^{\rho^{\prime}}\right) \times L\left(\langle n, d+1\rangle^{\rho^{\prime}}\right) \times \sigma^{\prime}$. 
But since both

$$
L\left(\langle n-1, d\rangle^{\rho^{\prime}}\right) \times L\left(\langle n+1, d\rangle^{\rho^{\prime}}\right) \quad \text { and } \quad L\left(\langle n, d-1\rangle^{\rho^{\prime}}\right) \times L\left(\langle n, d+1\rangle^{\rho^{\prime}}\right)
$$

have in their cuspidal supports $2 n d$ representations (counted with multiplicities), which is exactly the number of representations in the cuspidal support of $\pi$ (counted with multiplicities), we conclude that $\sigma^{\prime}=1$, as needed.

\section{On characters of irreducible unitary representations of $\operatorname{GL}(n, A)$}

5.1. The set of nonnegative integers is denoted by $\mathbb{Z}_{+}$. Fix $\rho \in \mathscr{C}_{F}$ and $\rho^{\prime} \in \mathscr{C}_{A}$. Let $R_{F}(\rho)$ be the subalgebra of $R_{F}$ generated by

$$
\left\{\delta\left(\left[v^{k_{1}} \rho, v^{k_{2}} \rho\right]\right) \mid k_{1}, k_{2} \in \frac{1}{2} \mathbb{Z}, k_{2}-k_{1} \in \mathbb{Z}_{+}\right\}
$$

and $R_{A}\left(\rho^{\prime}\right)$ the subalgebra of $R_{A}$ generated by

$$
\left\{\delta\left(\left[v_{\rho^{\prime}}^{k_{1}} \rho^{\prime}, v_{\rho^{\prime}}^{k_{2}} \rho^{\prime}\right]\right) \mid k_{1}, k_{2} \in \frac{1}{2} \mathbb{Z}, k_{2}-k_{1} \in \mathbb{Z}_{+}\right\} .
$$

Clearly, both algebras are polynomial over these sets of generators. Define an algebra isomorphism $\Psi_{\rho, \rho^{\prime}}: R_{F}(\rho) \rightarrow R_{A}^{\prime}\left(\rho^{\prime}\right)$ by

$$
\Psi_{\rho, \rho^{\prime}}: \delta\left(\left[v^{k_{1}} \rho, v^{k_{2}} \rho\right]\right) \mapsto \delta\left(\left[v_{\rho^{\prime}}^{k_{1}} \rho^{\prime}, v_{\rho^{\prime}}^{k_{2}} \rho^{\prime}\right]\right)
$$

for all $k_{1}, k_{2} \in \frac{1}{2} \mathbb{Z}, k_{2}-k_{1} \in \mathbb{Z}_{+}$.

Lemma. If we assume (U0), then

$$
\Psi_{\rho, \rho^{\prime}}:\left(L\left(\langle n, d\rangle^{v^{k} \rho}\right)=L\left(\langle n, d\rangle^{v^{k} \rho^{\prime} \rho^{\prime}}\right)\right.
$$

for all $n, d \in \mathbb{N}$ and $k \in \frac{1}{2} \mathbb{Z}$.

Proof. We work by induction on $n$. For $n=1$ (and all $d$ ) the lemma holds by definition of $\Psi_{\rho, \rho^{\prime}}$ (see $\S 1.14$ and §2.6). Fix $n \geq 1$ and assume that the formula of the lemma holds for all $n^{\prime} \leq n$. Applying $\Psi_{\rho, \rho^{\prime}}$ to the formula of the theorem in $\S 1.15$ (with $\nu^{k} \rho$ instead of $\rho$ ) and using the inductive assumption we get

$$
\begin{aligned}
& v_{\rho^{\prime}}^{1 / 2} L\left(\langle n, d\rangle^{v_{\rho^{\prime}}^{k} \rho^{\prime}}\right) \times v_{\rho^{\prime}}^{-1 / 2} L\left(\langle n, d\rangle^{v_{\rho^{\prime}}^{k} \rho^{\prime}}\right) \\
& =\Psi_{\rho, \rho^{\prime}}\left(L\left(\langle n+1, d\rangle^{v^{k} \rho}\right)\right) \times L\left(\langle n-1, d\rangle^{v_{\rho^{\prime}}^{k} \rho^{\prime}}\right)+L\left(\langle n, d+1\rangle^{v_{\rho^{\prime}}^{k} \rho^{\prime}}\right) \times L\left(\langle n, d-1\rangle^{v_{\rho^{\prime}}^{k} \rho^{\prime}}\right) .
\end{aligned}
$$

Subtracting this from the formula of the proposition in $\S 4.1$ (with $v_{\rho^{\prime}}^{k} \rho^{\prime}$ instead of $\rho^{\prime}$ in the formula) and using the fact that $R_{A}$ is an integral domain, one gets $\Psi_{\rho, \rho^{\prime}}\left(L\left(\langle n+1, d\rangle^{\nu^{k} \rho}\right)=L\left(\langle n+1, d\rangle^{\nu^{\prime} \rho^{\prime} \rho^{\prime}}\right)\right.$, as needed.

5.2. From the preceding lemma and the theorem in $\S 1.15$, we immediately get: 
Proposition. Assume that (U0) holds. Let $\rho^{\prime} \in \mathscr{C}_{A}$ and $n, d \in \mathbb{Z}, n, d \geq 1$. Let $W_{n}$ be the group of permutations of the set $\{1,2, \ldots, n\}$. Set $W_{n}^{(d)}=\left\{w \in W_{n} ; w(i)+d \geq i\right.$ for all $1 \leq i \leq n\}$. Then we have in $R_{A}$

$$
\begin{aligned}
L\left(\left(\left[v_{\rho^{\prime}} \rho^{\prime}, v_{\rho^{\prime}}^{d} \rho^{\prime}\right],\left[v_{\rho^{\prime}}^{2} \rho^{\prime}, v_{\rho^{\prime}}^{d+1} \rho^{\prime}\right], \ldots,\left[v_{\rho^{\prime}}^{n} \rho^{\prime}, v_{\rho^{\prime}}^{d+n-1} \rho^{\prime}\right]\right)\right) & \\
& =\sum_{w \in W_{n}^{(d)}}(-1)^{w} \prod_{i=1}^{n} \delta\left(\left[v_{\rho^{\prime}}^{i} \rho^{\prime}, v_{\rho^{\prime}}^{w(i)+(d-1)} \rho^{\prime}\right]\right) .
\end{aligned}
$$

\section{Jacquet-Langlands correspondence}

6.1. A. I. Badulescu [2002] studied very natural extensions of Jacquet-Langlands correspondences. We recall here some of his considerations (in a slightly different notation).

In $\$ 2.3$ we recalled the Jacquet-Langlands correspondences

$$
\mathrm{JL}_{p}: \mathscr{D}(\mathrm{GL}(p, A)) \rightarrow \mathscr{D}\left(\mathrm{GL}\left(p d_{A}, F\right)\right),
$$

which are uniquely determined by the requirement that the characters $\Theta_{\delta^{\prime}}$ and $\Theta_{\mathrm{JL}_{p}\left(\delta^{\prime}\right)}$ satisfy

$$
(-1)^{p} \Theta_{\delta^{\prime}}\left(g^{\prime}\right)=(-1)^{p d_{A}} \Theta_{\mathrm{JL}_{p}\left(\delta^{\prime}\right)}(g)
$$

whenever $g^{\prime}$ and $g$ have the same characteristic polynomials, and when this polynomial is separable.

The correspondences above are bijections, so instead of the correspondences $\mathrm{JL}_{p}$, we could consider their inverses $\mathrm{JL}_{p}^{-1}$.

The mappings $\mathrm{JL}_{p}, p \geq 1$, define in a natural way an injective mapping

$$
\mathrm{JL}: \mathscr{D}_{A} \rightarrow \mathscr{D}_{F} \text {. }
$$

Since the algebras $R_{A}$ and $R_{F}$ are polynomial over $\mathscr{D}_{A}$ and $\mathscr{D}_{F}$ respectively, $\mathrm{JL}_{p}$ can be uniquely extended to a ring homomorphism of $R_{A}$ into $R_{F}$, which will be again denoted by

$$
\mathrm{JL}: R_{A} \rightarrow R_{F}
$$

Clearly, the extension is also injective.

The homomorphism JL carries $\operatorname{Groth} \operatorname{GL}(p, A)$ to $\operatorname{Groth} \operatorname{GL}\left(p d_{A}, F\right)$, and we denote this restriction again by

$$
\mathrm{JL}_{p}: \text { Groth } \mathrm{GL}(p, A) \rightarrow \text { Groth } \mathrm{GL}\left(p d_{A}, F\right) .
$$

Then this extended $\mathrm{JL}_{p}$ again satisfies the relation

$$
(-1)^{p} \Theta_{\pi^{\prime}}\left(\pi^{\prime}\right)=(-1)^{p d_{A}} \Theta_{\mathrm{JL}_{p}\left(\pi^{\prime}\right)}(g)
$$

for any $\pi \in \operatorname{GL}(p, A)^{\sim}$. 
Let

$$
\mathscr{D}_{F}^{\left(d_{A}\right)}=\bigcup_{p \geq 1} \mathscr{D}\left(\mathrm{GL}\left(p d_{A}, F\right)\right) .
$$

Then JL defines a bijection of $\mathscr{D}_{A}$ onto $\mathscr{D}_{F}^{\left(d_{A}\right)}$. Denote the inverse mapping by

$$
\mathrm{LJ}: \mathscr{D}_{F}^{\left(d_{A}\right)} \rightarrow \mathscr{D}_{A} .
$$

There exists a unique ring homomorphism $R_{F} \rightarrow R_{A}$ which extends LJ and which sends all the elements from $\mathscr{D}_{F} \backslash \mathscr{D}_{F}^{\left(d_{A}\right)}$ to $0 \in R_{A}$. This extension will be denoted again by

$$
\mathrm{LJ}: R_{F} \rightarrow R_{A} \text {. }
$$

If $d_{A} \mid m$, we denote by $\mathrm{LJ}_{m}$ the restriction

$$
\mathrm{LJ}_{m}: \text { Groth } \mathrm{GL}(m, F) \rightarrow \operatorname{Groth} \mathrm{GL}\left(m / d_{A}, A\right) .
$$

Otherwise, we take (formally) $\mathrm{LJ}_{m}=0$ (as a mapping from Groth $\mathrm{GL}(m, F)$ into $\left.R_{A}\right)$.

Let $I_{F, A}$ be the ideal in $R_{F}$ generated by $\mathscr{D}_{F} \backslash \mathscr{D}_{F}^{\left(d_{A}\right)}$ (clearly, this ideal is graded). This is just the kernel of LJ. Therefore,

$$
R_{A} \cong R_{F} / I_{F, A} .
$$

Further, suppose that $\varphi \in \operatorname{Groth} \operatorname{GL}(m, F)$ is in $I_{F, A}$ and $d_{A} \mid m$. Then for regular semisimple $g \in \operatorname{GL}(m, F)$ we have $\Theta_{\varphi}(g)=0$, where $\Theta_{\varphi}$ denotes the formal character of $\varphi$. Therefore,

$$
(-1)^{m} \Theta_{\varphi}(g)=(-1)^{m / d_{A}} \Theta_{\mathrm{LJ}(\varphi)}\left(g^{\prime}\right)
$$

whenever $g$ and $g^{\prime}$ have the same characteristic polynomials, and when this polynomial is separable. Clearly,

$$
\mathrm{LJ} \circ \mathrm{JL}=\mathrm{id}_{R_{A}} .
$$

The correspondence JL: $R_{A} \rightarrow R_{F}$, which we considered first, does not behave well with respect to irreducibility. Namely, one sees easily (as in the comments after [Badulescu 2002, Theorem 3.1]) that JL does not in general carry irreducible representations to irreducible ones (up to a sign). A similar situation happens with unitarity. In general even irreducible unitary representations are carried neither to irreducible unitary representations (up to a sign), nor to linear combinations of irreducible unitary representations.

Assuming (U0) holds, we shall see in the rest of the paper that the correspondence LJ: $R_{F} \rightarrow R_{A}$ behave well with respect to irreducible unitary representations, i.e., that it carries such representations either again to the irreducible unitary representations (up to a sign) or to 0 . 
6.2. Let $\rho^{\prime} \in \mathscr{C}_{A}$. Suppose that $\delta\left(\left[\rho, v^{s_{\rho^{\prime}}-1} \rho\right]\right) \in \mathscr{D}_{F}$ corresponds to $\rho^{\prime}$ under the Jacquet-Langlands correspondence (here $\left.\rho \in \mathscr{C}_{F}\right)$. Then $\delta\left(\left[\rho, v^{s_{\rho^{\prime}}-1} \rho\right]\right)$ corresponds to $\delta\left(\left[\rho^{\prime}, v_{\rho^{\prime}}^{k-1} \rho^{\prime}\right]\right)$.

6.3. Fix an irreducible cuspidal representation $\rho$ of $\operatorname{GL}(m, F)$. Let $s^{\prime} m$ be the smallest common multiple of $m$ and $d_{A}$. The fact $s^{\prime} m \mid d_{A} m$ implies

$$
s^{\prime} \mid d_{A} .
$$

Note that $\delta\left(\left[\rho, v^{s^{\prime}-1} \rho\right]\right)$ is an irreducible essentially square-integrable representation of $\mathrm{GL}\left(s^{\prime} m, F\right)$. Therefore, it lifts under the Jacquet-Langlands correspondence to an irreducible essentially square-integrable representation $\rho^{\prime}$ in $R_{A}$. A short discussion implies that $\rho^{\prime}$ is cuspidal, and then $s_{\rho^{\prime}}=s^{\prime}$.

Now $\rho^{\prime}$ is a representation of $\operatorname{GL}(p, A)$, where $p=m s_{\rho^{\prime}} / d_{A}$. Since $s^{\prime} m$ is the smallest common multiple of $m$ and $d_{A}$, this implies $\left(p, s_{\rho^{\prime}}\right)=1$ (if $k$ were the greatest common divisor, then $k d_{A}$ and $k m$ would divide $s^{\prime} m$ ). Further, the smallest common multiple of $d_{A}$ and $p d_{A} / s_{\rho^{\prime}}=m$ is $s_{\rho^{\prime}} m=p d_{A}$.

6.4. Assuming (U0) we shall compute $\mathrm{LJ}(\pi)$ for irreducible unitary representations of general linear groups over the field. Since LJ is a ring homomorphism, for this it will be enough to compute

$$
\mathrm{LJ}\left(L\left(\langle r, d\rangle^{\rho}\right) \in R_{A}, \quad r, d \in \mathbb{N} .\right.
$$

Suppose that we are in the situation of $\S 6.3$, and suppose that $s_{\rho^{\prime}}=1$ (which means that $\rho$ corresponds to $\rho^{\prime}$ under the Jacquet-Langlands correspondence). Then the theorem in $\$ 1.15$ and the proposition in $\$ 5.2$ directly imply

$$
\mathrm{LJ}\left(L\left(\langle r, d\rangle^{\rho}\right)\right)=L\left(\langle r, d\rangle^{\rho^{\prime}}\right) .
$$

It remains therefore to consider the case

$$
s_{\rho^{\prime}} \geq 2 \text {. }
$$

We shall assume this in the rest of the paper.

If $r=1$, then we know $\operatorname{LJ}\left(L\left(\langle 1, d\rangle^{\rho}\right)\right)$ by $\S 6.2$, so we can assume also $r \geq 2$. To simplify the notation, we shall often denote below $s_{\rho^{\prime}}$ by $n$ :

$$
s_{\rho^{\prime}}=n \text {. }
$$

\section{Calculation of the Jacquet-Langlands correspondence in the unitary case}

\section{I: the case $r \leq d$ and $s_{\rho^{\prime}} \mid d$}

7.1. In this section, we assume $r \leq d$ and $s_{\rho^{\prime}} \mid d$ (i.e., $n \mid d$ ).

We shall use below the following technical lemma, identical to [Tadic 1996, Lemma 3.1], whose statement we repeat here for convenience. 
Denote by $W_{r}^{\prime}$ the group of permutations of $\{0,1, \ldots, r-1\}$. The sign of a permutation $w$ will be denoted by $(-1)^{w}$.

Lemma. Write $r=a n+b$, with $a, b \in \mathbb{Z}$ such that $0 \leq b \leq n-1$.

(i) Let

$$
W_{r}^{\prime}(n)=\left\{w \in W_{r}^{\prime} ; n \mid(w(i)-i) \text { for all } 0 \leq i \leq r-1\right\} .
$$

For $0 \leq \ell \leq \min (n, r)-1$, set

$$
W_{r}^{\prime}(n ; \ell)=\left\{w \in W_{r}^{\prime}(n) ; w(i)=i \text { if } n \nmid(i-\ell)\right\} .
$$

Then $W_{r}^{\prime}(n)$ is a subgroup of $W_{r}^{\prime}, W_{r}^{\prime}(n ; \ell)$ are subgroups of $W_{r}^{\prime}(n)$ and $W_{r}^{\prime}(n)$ is a direct product of $W_{r}^{\prime}(n ; \ell)$, for $\ell=0,1,2, \ldots, \min (n, r)-1$.

(ii) Let $0 \leq \ell \leq b-1$ (resp. $b \leq \ell \leq \min (n, r)-1)$. For $w \in W_{a+1}^{\prime}$ (resp. $w \in W_{a}^{\prime}$ ) define $w^{*} \in W_{r}^{\prime}$ by

$$
w^{*}(j)= \begin{cases}j & \text { if } n \nmid(i-\ell), \\ \ell+n w(i) & \text { if } j=\ell+n i .\end{cases}
$$

Then $w \mapsto w^{*}$ is an isomorphism of $W_{a+1}^{\prime}\left(\right.$ resp. $\left.W_{a}^{\prime}\right)$ onto $W_{r}^{\prime}(n ; \ell)$. Further, $(-1)^{w}=(-1)^{w^{*}}$.

7.2. Let

$$
\Pi=L\left(\left(\left[\rho, v^{d-1} \rho\right],\left[v \rho, v^{d} \rho\right], \ldots,\left[v^{r-1} \rho, v^{r-1+d-1} \rho\right]\right)\right) .
$$

We now start the computation of

$$
\begin{aligned}
\mathrm{LJ}(\Pi) & =\mathrm{LJ}\left(L\left(\left(\left[\rho, v^{d-1} \rho\right],\left[v \rho, v^{d} \rho\right], \ldots,\left[v^{r-1} \rho, v^{r-1+d-1} \rho\right]\right)\right)\right) \\
& =\sum_{w \in W_{r}^{\prime}}(-1)^{w} \prod_{i=0}^{r-1} \operatorname{LJ}\left(\delta\left(\left[v^{i} \rho, v^{w(i)+(d-1)} \rho\right]\right)\right) \\
& =\sum_{w \in W_{r}^{\prime}(n)}(-1)^{w} \prod_{i=0}^{r-1} \operatorname{LJ}\left(\delta\left(\left[v^{i} \rho, v^{w(i)+(d-1)} \rho\right]\right)\right),
\end{aligned}
$$

where we have used the fact that $n \mid d$ and $r \leq d$.

Write $r=a n+b$ with $a, b \in \mathbb{Z}$ and $0 \leq b \leq n-1$. We use the preceding lemma to modify the sum:

$$
\operatorname{LJ}(\Pi)=\sum_{w \in W_{r}^{\prime}(n)}(-1)^{w} \prod_{i=0}^{r-1} \operatorname{LJ}\left(\delta\left(\left[v^{i} \rho, v^{w(i)+(d-1)} \rho\right]\right)\right)
$$




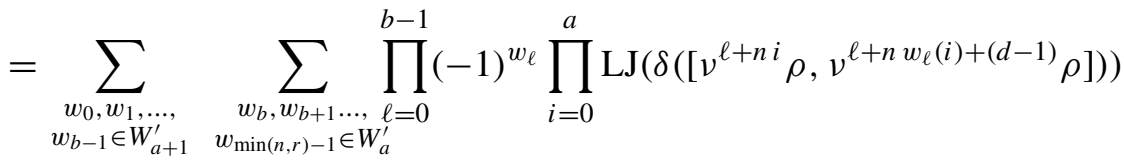

$$
\begin{aligned}
& \times \prod_{\ell=b}^{\min (n, r)-1}(-1)^{w_{\ell}} \prod_{i=0}^{a-1} \mathrm{LJ}\left(\delta\left(\left[v^{\ell+n i} \rho, v^{\ell+n w_{\ell}(i)+(d-1)} \rho\right]\right)\right)
\end{aligned}
$$

$$
\begin{gathered}
=\prod_{\ell=0}^{b-1} \sum_{w_{\ell} \in W_{a+1}^{\prime}}(-1)^{w_{\ell}} \prod_{i=0}^{a} \operatorname{LJ}\left(\delta\left(\left[v^{\ell+n i} \rho, v^{\ell+n w_{\ell}(i)+(d-1)} \rho\right]\right)\right) \\
\quad \times \prod_{\ell=b}^{\min (n, r)-1} \sum_{w_{\ell} \in W_{a}^{\prime}}(-1)^{w_{\ell}} \prod_{i=0}^{a-1} \operatorname{LJ}\left(\delta\left(\left[v^{\ell+n i} \rho, v^{\ell+n w_{\ell}(i)+(d-1)} \rho\right]\right)\right) .
\end{gathered}
$$

Now assume that $\delta\left(\left[\rho, v^{n-1} \rho\right]\right)$ and $\rho^{\prime}$ correspond under the Jacquet-Langlands correspondence as in $\$ 6.2$. Then

$$
\begin{aligned}
& \mathrm{LJ}(\Pi)=\left(\prod_{\ell=0}^{b-1} \sum_{w_{\ell} \in W_{a+1}^{\prime}}(-1)^{w_{\ell}} \prod_{i=0}^{a} \delta\left(\left[v^{\ell+n i} \rho^{\prime}, v^{\ell+n i} v_{\rho^{\prime}}^{w_{\ell}(i)-i+(d / n-1)} \rho^{\prime}\right]\right)\right. \\
& \times \prod_{\ell=b}^{\min (n, r)-1} \sum_{w_{\ell} \in W_{a}^{\prime}}(-1)^{w_{\ell}} \prod_{i=0}^{a-1} \delta\left(\left[v^{\ell+n i} \rho^{\prime}, v^{\ell+n i} v_{\rho^{\prime}}^{w_{\ell}(i)-i+(d / n-1)} \rho^{\prime}\right]\right) \\
& =\left(\prod_{\ell=0}^{b-1} v_{\rho^{\prime}}^{\ell / n} \sum_{w_{\ell} \in W_{a+1}^{\prime}}(-1)^{w_{\ell}} \prod_{i=0}^{a} \delta\left(\left[v_{\rho^{\prime}}^{i} \rho^{\prime}, v_{\rho^{\prime}}^{w_{\ell}(i)+(d / n-1)} \rho^{\prime}\right]\right)\right. \\
& \times \prod_{\ell=b}^{\min (n, r)-1} v_{\rho^{\prime}}^{\ell / n} \sum_{w_{\ell} \in W_{a}^{\prime}}(-1)^{w_{\ell}} \prod_{i=0}^{a-1} \delta\left(\left[v_{\rho^{\prime}}^{i} \rho^{\prime}, v_{\rho^{\prime}}^{w_{\ell}(i)+(d / n-1)} \rho^{\prime}\right]\right) .
\end{aligned}
$$

Note that $r \leq d$ implies $r-b \leq d$, which implies $a \leq d / n$.

If $b \geq 1$, then $r-b<d$, which implies $a<d / n$ and further $a+1 \leq d / n$ (since $n \mid d)$. Therefore

$$
\begin{aligned}
& \mathrm{LJ}(\Pi)=\prod_{\ell=0}^{b-1} v_{\rho^{\prime}}^{\ell / n} L\left(\left(\left[\rho^{\prime}, v_{\rho^{\prime}}^{d / n-1} \rho^{\prime}\right],\left[v_{\rho^{\prime}} \rho^{\prime}, v_{\rho^{\prime}}^{d / n} \rho^{\prime}\right], \ldots,\left[v_{\rho^{\prime}}^{a} \rho^{\prime}, v_{\rho^{\prime}}^{a+d / n-1} \rho^{\prime}\right]\right)\right) \\
& \quad \times \prod_{\ell=b}^{\min (n, r)-1} v_{\rho^{\prime}}^{\ell / n} L\left(\left(\left[\rho^{\prime}, v_{\rho^{\prime}}^{d / n-1} \rho^{\prime}\right],\left[v_{\rho^{\prime}} \rho^{\prime}, v_{\rho^{\prime}}^{d / n} \rho^{\prime}\right], \ldots,\left[v_{\rho^{\prime}}^{a-1} \rho^{\prime}, v_{\rho^{\prime}}^{a-1+d / n-1} \rho^{\prime}\right]\right)\right) .
\end{aligned}
$$


7.3. Now suppose that $\rho$ is unitary and that $\delta\left(\rho, s_{\rho^{\prime}}\right)=\delta(\rho, n)$ corresponds to $\rho^{\prime \prime}$ under the Jacquet-Langlands correspondence. Then $\rho^{\prime \prime}$ is unitary. Further

$$
\begin{aligned}
\rho^{\prime \prime} & =\mathrm{LJ}(\delta(\rho, n))=\mathrm{LJ}\left(v^{-(n-1) / 2} \delta\left(\left[\rho, v^{n-1} \rho\right]\right)\right)=v^{-(n-1) / 2} \mathbf{L J}\left(\delta\left(\left[\rho, v^{n-1} \rho\right]\right)\right) \\
& =v_{\rho^{\prime}}^{-(n-1) /(2 n)} \rho^{\prime} .
\end{aligned}
$$

Note that $v_{\rho^{\prime}}=v_{\rho^{\prime \prime}}$.

Now we compute (for $r \leq d$ and $n \mid d$ )

$\operatorname{LJ}\left(L\left(\langle r, d\rangle^{\rho}\right)\right)$

$$
\begin{gathered}
=\operatorname{LJ}\left(v^{-\frac{r+d}{2}+1} L\left(\left(\left[\rho, v^{d-1} \rho\right],\left[v \rho, v^{d} \rho\right], \ldots,\left[v^{r-1} \rho, v^{r-1+d-1} \rho\right]\right)\right)\right) \\
=v^{-\frac{r+d}{2}+1} \prod_{\ell=0}^{b-1} v_{\rho^{\prime}}^{\frac{\ell}{n}} v_{\rho^{\prime}}^{\frac{a+1+d / n}{2}-1} L\left(\langle a+1, d / n\rangle^{\rho^{\prime}}\right) \\
\times \prod_{\ell=b}^{\min (n, r)-1} v_{\rho^{\prime}}^{\frac{\ell}{n}} v_{\rho^{\prime}}^{\frac{a+d / n}{2}-1} L\left(\langle a, d / n\rangle^{\rho^{\prime}}\right) .
\end{gathered}
$$

Since

$$
v^{-\frac{r+d}{2}+1}=v_{\rho^{\prime}}^{\frac{-r-d+2}{2 n}}=v_{\rho^{\prime}}^{\frac{-a n-b-d+2}{2 n}},
$$

we have

$$
\begin{aligned}
& \mathrm{LJ}\left(L\left(\langle r, d\rangle^{\rho}\right)\right) \\
& =\prod_{\ell=0}^{b-1} v_{\rho^{\prime}}^{\frac{-b+2+2 \ell-n}{2 n}} L\left(\langle a+1, d / n\rangle^{\rho^{\prime}}\right) \prod_{\ell=b}^{\min (n, r)-1} v_{\rho^{\prime}}^{\frac{-b+2+2 \ell-2 n}{2 n}} L\left(\langle a, d / n\rangle^{\rho^{\prime}}\right) \\
& =\prod_{\ell=0}^{b-1} v_{\rho^{\prime}}^{\frac{-b+2+2 \ell-n}{2 n}} L\left(\langle a+1, d / n\rangle^{v^{\frac{n-1}{2 n}} \rho^{\prime \prime}}\right) \\
& \times \prod_{\ell=b}^{\min (n, r)-1} v_{\rho^{\prime}}^{\frac{-b+2+2 \ell-2 n}{2 n}} L\left(\langle a, d / n\rangle^{v_{\rho^{\prime}}^{\frac{n-1}{2 n}} \rho^{\prime \prime}}\right) \\
& =\prod_{\ell=0}^{b-1} v_{\rho^{\prime \prime}}^{\frac{-b+1+2 \ell}{2 n}} L\left(\langle a+1, d / n\rangle^{\rho^{\prime \prime}}\right) \prod_{\ell=b}^{\min (n, r)-1} v_{\rho^{\prime \prime}}^{\frac{-b+1+2 \ell-n}{2 n}} L\left(\langle a, d / n\rangle^{\rho^{\prime \prime}}\right) \\
& =\prod_{\ell^{\prime}=-\frac{b-1}{2}}^{\frac{b-1}{2}} v_{\rho^{\prime \prime}}^{\frac{\ell^{\prime}}{n}} L\left(\langle a+1, d / n\rangle^{\rho^{\prime \prime}}\right) \prod_{\ell=b}^{\min (n, r)-1} v_{\rho^{\prime \prime}}^{\frac{(-b-n+1) / 2+\ell}{n}} L\left(\langle a, d / n\rangle^{\rho^{\prime \prime}}\right) .
\end{aligned}
$$

For an irreducible representation $\pi$ of $\operatorname{GL}(l, A)$, positive integer $l$ and a nonnegative integer $k$, define the string

$$
\operatorname{str}_{v_{\rho^{\prime}}}(k, l, \pi)
$$


as

$$
\left(v_{\rho^{\prime}}^{\frac{-(k-1) / 2}{l}} \pi\right) \times\left(v_{\rho^{\prime}}^{\frac{-(k-1) / 2+1}{l}} \pi\right) \times\left(v_{\rho^{\prime}}^{\frac{-(k-1) / 2+2}{l}} \pi\right) \times \cdots \times\left(v_{\rho^{\prime}}^{\frac{(k-1) / 2}{l}} \pi\right) .
$$

If $k=0$, we take the string to be the identity of $R_{A}$ (i.e., the trivial representation of the trivial group $\mathrm{GL}(0, A))$.

The next result follows directly from this:

Proposition. Suppose that (U0) holds. Assume that $\delta(\rho, n)$ corresponds to $\rho^{\prime \prime} \in \mathscr{C}_{A}$ under the Jacquet-Langlands correspondence. Let

$$
r \leq d, \quad n \mid d, \quad 1 \leq n
$$

Write

$$
r=a n+b, \quad a, b \in \mathbb{Z}, \quad 0 \leq b \leq n-1 .
$$

Then

$$
\begin{aligned}
L J\left(L\left(\langle r, d\rangle^{\rho}\right)\right)=\operatorname{str}_{v_{\rho^{\prime \prime}}}(b, n, L(\langle a+ & \left.\left.1, d / n\rangle^{\rho^{\prime \prime}}\right)\right) \\
& \times \operatorname{str}_{v_{\rho^{\prime \prime}}}\left(\min (n, r)-b, n, L\left(\langle a, d / n\rangle^{\rho^{\prime \prime}}\right)\right) .
\end{aligned}
$$

Remark. Our definition of $\operatorname{str}_{v_{\rho^{\prime}}}(k, l, \pi)$ is more general than we need here. In this paper, whenever we use $\operatorname{str}_{v_{\rho^{\prime}}}(k, l, \pi)$, we have $l=s_{\rho^{\prime}}=n$. Then $\operatorname{str}_{v_{\rho^{\prime}}}(k, n, \pi)$, which equals $\operatorname{str}_{v}(k, 1, \pi)$ in this case, will be denoted simply by

$$
\operatorname{str}(k, \pi)
$$

and we have

$$
\operatorname{str}(k, \pi)=\left(v^{-(k-1) / 2} \pi\right) \times\left(v^{-(k-1) / 2+1} \pi\right) \times\left(v^{-(k-1) / 2+2} \pi\right) \times \cdots \times\left(v^{(k-1) / 2} \pi\right),
$$

with neither $v_{\rho^{\prime}}$ nor $n=s_{\rho^{\prime}}$ showing up on the right-hand side.

We shall use both notations, $\operatorname{str}(k, \pi)$ and $\operatorname{str}_{v_{\rho^{\prime}}}(k, n, \pi)$. The later will be convenient when we study unitarity.

Now the proposition above can be rewritten:

Proposition'. With the same assumptions as in the preceding proposition, we have $\operatorname{LJ}\left(L\left(\langle r, d\rangle^{\rho}\right)\right)=\operatorname{str}\left(b, L\left(\langle a+1, d / n\rangle^{\rho^{\prime \prime}}\right)\right) \operatorname{str}\left(\min (n, r)-b, L\left(\langle a, d / n\rangle^{\rho^{\prime \prime}}\right)\right)$.

We can rewrite this formula as

$$
\begin{aligned}
\operatorname{LJ}\left(L\left(\langle r, d\rangle^{\rho}\right)\right)=\operatorname{str}(r-[r / n] n & \left., L\left(\langle[r / n]+1, d / n\rangle^{\rho^{\prime \prime}}\right)\right) \\
& \times \operatorname{str}\left(\min (n, r)-r+[r / n] n, L\left(\langle[r / n], d / n\rangle^{\rho^{\prime \prime}}\right)\right),
\end{aligned}
$$

where $[x]$ denotes the greatest integer which does not exceed $x$. 


\section{Unitarity of the Jacquet-Langlands correspondence of irreducible unitary representations, I}

8.1. We now show that $\operatorname{LJ}\left(L\left(\langle r, d\rangle^{\rho}\right)\right)$, which we have computed in the previous section, is irreducible and unitary if $\rho \in \mathscr{C}_{F}$ is unitary. After this, we shall show that $\operatorname{LJ}\left(v^{\beta}\left(L\left(\langle r, d\rangle^{\rho}\right)\right) \times v^{-\beta}\left(L\left(\langle r, d\rangle^{\rho}\right)\right)\right)$ is unitary for $0<\beta<\frac{1}{2}$.

If $b=0$, then $\operatorname{str}_{v_{\rho^{\prime \prime}}}\left(b, n, L\left(\langle a+1, d / n\rangle^{\rho^{\prime \prime}}\right)\right)$ is irreducible unitary since it equals 1. Suppose $b \geq 1$. Then $0 \leq(b-1) /(2 n)<n /(2 n)=\frac{1}{2}$, and we conclude that $\operatorname{str}_{v_{\rho^{\prime \prime}}}\left(b, n, L\left(\langle a+1, d / n\rangle^{\rho^{\prime \prime}}\right)\right)$ is again irreducible unitary.

If $r<n$, then $\operatorname{str}_{v_{\rho^{\prime \prime}}}\left(\min (n, r)-b, n, L\left(\langle a, d / n\rangle^{\rho^{\prime \prime}}\right)\right)$ is irreducible unitary since it equals 1 . Suppose $r \geq n$. Then

$$
\operatorname{str}_{v_{\rho^{\prime \prime}}}\left(\min (n, r)-b, n, L\left(\langle a, d / n\rangle^{\rho^{\prime \prime}}\right)\right)=\operatorname{str}_{v_{\rho^{\prime \prime}}}\left(n-b, n, L\left(\langle a, d / n\rangle^{\rho^{\prime \prime}}\right)\right) .
$$

Since now $0 \leq(n-b-1) /(2 n) \leq(n-1) /(2 n)<\frac{1}{2}$, this implies that, once more, $\operatorname{str}_{v_{\rho^{\prime \prime}}}\left(\min (n, r)-b, n, L\left(\langle a, d / n\rangle^{\rho^{\prime \prime}}\right)\right.$ is irreducible unitary. Thus $\operatorname{LJ}\left(L\left(\langle r, d\rangle^{\rho}\right)\right)$ is irreducible unitary.

For $0<\beta<\frac{1}{2}$, set

$$
\pi\left(L\left(\langle r, d\rangle^{\rho}\right), \beta\right)=v^{\beta} L\left(\langle r, d\rangle^{\rho}\right) \times v^{-\beta} L\left(\langle r, d\rangle^{\rho}\right) .
$$

We show that $\operatorname{LJ}\left(\pi\left(L\left(\langle r, d\rangle^{\rho}\right), \beta\right)\right)$ is irreducible unitary. Observe that

$$
\begin{aligned}
& \operatorname{LJ}\left(\pi\left(L\left(\langle r, d\rangle^{\rho}\right), \beta\right)\right) \\
& =v^{\beta} \operatorname{LJ}\left(L\left(\langle r, d\rangle^{\rho}\right)\right) v^{-\beta} \operatorname{LJ}\left(L\left(\langle r, d\rangle^{\rho}\right)\right) \\
& =v_{\rho^{\prime \prime}}^{\beta / n} \operatorname{str}_{v_{\rho^{\prime \prime}}}\left(b, n, L\left(\langle a+1, d / n\rangle^{\rho^{\prime \prime}}\right)\right) \times v_{\rho^{\prime \prime}}^{-\beta / n} \operatorname{str}_{\nu_{\rho^{\prime \prime}}}\left(b, n, L\left(\langle a+1, d / n\rangle^{\rho^{\prime \prime}}\right)\right) \\
& \quad \times v_{\rho^{\prime \prime}}^{\beta / n} \operatorname{str}_{v_{\rho^{\prime \prime}}}\left(\min (n, r)-b, n, L\left(\langle a, d / n\rangle^{\rho^{\prime \prime}}\right)\right) \\
& \quad \times v_{\rho^{\prime \prime}}^{-\beta / n} \operatorname{str}_{v_{\rho^{\prime \prime}}}\left(\min (n, r)-b, n, L\left(\langle a, d / n\rangle^{\rho^{\prime \prime}}\right)\right) .
\end{aligned}
$$

This implies that it is enough to show unitarity (and irreducibility, which follows from unitarity) for

$$
v_{\rho^{\prime \prime}}^{\beta / n} \operatorname{str}_{v_{\rho^{\prime \prime}}}\left(b, n, L\left(\langle a+1, d / n\rangle^{\rho^{\prime \prime}}\right)\right) \times v_{\rho^{\prime \prime}}^{-\beta / n} \operatorname{str}_{v_{\rho^{\prime \prime}}}\left(b, n, L\left(\langle a+1, d / n\rangle^{\rho^{\prime \prime}}\right)\right)
$$

if $b \geq 1$, and for

$$
v_{\rho^{\prime \prime}}^{\beta / n} \operatorname{str}_{v_{\rho^{\prime \prime}}}\left(n-b, n, L\left(\langle a, d / n\rangle^{\rho^{\prime \prime}}\right)\right) \times v_{\rho^{\prime \prime}}^{-\beta / n} \operatorname{str}_{v_{\rho^{\prime \prime}}}\left(n-b, n, L\left(\langle a, d / n\rangle^{\rho^{\prime \prime}}\right)\right)
$$

if $r \geq n$. For the first representation, we need to show that $0 \leq(b-1) /(2 n)+\beta / n<\frac{1}{2}$ if $b \geq 1$; for the second, that $0 \leq(n-b-1) /(2 n)+\beta / n<\frac{1}{2}$ if $r \geq n$. This obviously holds since $\beta<\frac{1}{2}$. 


\section{Calculation of the Jacquet-Langlands correspondence in the unitary case II: the case $r \leq d$ and $s_{\rho^{\prime}}$ not dividing $d$}

9.1. In this section we assume that $r \leq d$ and $s_{\rho^{\prime}} \nmid d$ (i.e., $n \nmid d$ ).

If $n \nmid r d$, then one sees directly that

$$
\mathrm{LJ}\left(L\left(\left(\left[\rho, v^{d-1} \rho\right],\left[v \rho, v^{d} \rho\right], \ldots,\left[v^{r-1} \rho, v^{r-1+d-1} \rho\right]\right)\right)\right)=0 .
$$

Therefore, we need only to consider the case $n \mid r d$. Soon we shall see that a stronger assumption needs to be imposed to get a nonzero result.

Write $r=a n+b$, with $a, b \in \mathbb{Z}$ and $0 \leq b \leq n-1$. Now $n \mid r d$ implies $n \mid b d$. We continue to assume

$$
n \geq 2 \text {. }
$$

If $r=1$, then $\operatorname{LJ}\left(L\left(\left(\left[\rho, v^{d-1} \rho\right]\right)\right)\right)=0$ since $n \nmid d$. Therefore, we shall assume in the sequel that

$$
r \geq 2 \text {. }
$$

9.2. The next result is a modification of [Tadić 1996, Lemma 4.1].

Lemma. Suppose $n \nmid d$. If the set

$$
\begin{aligned}
X_{r}(n, d) & =\left\{w \in W_{r}^{\prime} ; n \mid(d+w(i)-i) \text { for all } 0 \leq i \leq r-1\right\} \\
& =\left\{w \in W_{r}^{\prime} ; n \mid\left(d+i-w^{-1}(i)\right) \text { for all } 0 \leq i \leq r-1\right\}
\end{aligned}
$$

is nonempty, then $n \mid r$.

Proof. Suppose $X_{r}(n, d) \neq \varnothing$. Clearly, the identity is not in $X_{r}(n, d)$ since $n \nmid d$.

Take some $w \in X_{r}(n, d)$. Note that for $0 \leq i \leq r-2, n \mid(d+w(i)-i)$ and $n \mid(d+w(i+1)-i-1)$ imply $n \mid(w(i)-w(i+1)+1)$ Suppose $w(i)-w(i+1)+1=0$ for all $i$ as above. This implies $w(1)=w(0)+1, w(2)=w(0)+2, \ldots, w(r-2)=$ $w(0)+r-2$, which implies $w(0)=1$ (since $w$ cannot be identity). This implies $w(r-1)=0$.

Since $w \in X_{r}(n, d)$, we get $n \mid(d+1)$ and $n \mid(d+w(r-1)-(r-1))=(d+1-r)$. These two relations imply $n \mid r$.

Therefore it remains to consider the case when

$$
w(i)-w(i+1)+1 \neq 0
$$

for some $0 \leq i \leq r-2$. If $w(i)-w(i+1)+1$ is negative, then $w(i)-w(i+1)+1 \leq$ $-n$, which implies $w(i)+n+1 \leq w(i+1)$. This implies $n+1 \leq r-1$. If $w(i)-w(i+1)+1$ is positive, then $n \leq w(i)-w(i+1)+1$, which implies $n \leq r$. Thus we have proved (up to now) that

$$
n \leq r .
$$


We have written $r=a n+b$ with $a, b \in \mathbb{Z}$ and $0 \leq b \leq n-1$. We know that $a \geq 1$. Write

$$
d=c n+d^{\prime}, \quad \text { with } c, d^{\prime} \in \mathbb{Z} \text { and } 1 \leq d^{\prime} \leq n-1 \leq r-1,
$$

which is possible since $n \nmid d$.

Since the elements of $X_{r}(n, d) \subseteq W_{r}^{\prime}$ are bijections, for each $i \in\{0,1, \ldots, n-1\}$ we must have

$$
\begin{aligned}
\operatorname{card}(\{i+k n ; k \in \mathbb{Z}\} \cap[0, r-1]) & =\operatorname{card}(\{i-d+k n ; k \in \mathbb{Z}\} \cap[0, r-1]) \\
& =\operatorname{card}\left(\left\{i-d^{\prime}+k n ; k \in \mathbb{Z}\right\} \cap[0, r-1]\right) \\
& =\operatorname{card}\left(\{i+k n ; k \in \mathbb{Z}\} \cap\left[d^{\prime}, d^{\prime}+r-1\right]\right) .
\end{aligned}
$$

From this we get

$$
\begin{aligned}
& \operatorname{card}\left(\{i+k n ; k \in \mathbb{Z}\} \cap\left[0, d^{\prime}-1\right]\right)+\operatorname{card}\left(\{i+k n ; k \in \mathbb{Z}\} \cap\left[d^{\prime}, r-1\right]\right) \\
& \quad=\operatorname{card}\left(\{i+k n ; k \in \mathbb{Z}\} \cap\left[d^{\prime}, r-1\right]\right)+\operatorname{card}\left(\{i+k n ; k \in \mathbb{Z}\} \cap\left[r, d^{\prime}+r-1\right]\right) .
\end{aligned}
$$

Thus for each $i \in\{0,1, \ldots, n-1\}$ we have

$$
\operatorname{card}\left(\{i+k n ; k \in \mathbb{Z}\} \cap\left[0, d^{\prime}-1\right]\right)=\operatorname{card}\left(\{i+k n ; k \in \mathbb{Z}\} \cap\left[r, d^{\prime}+r-1\right]\right) .
$$

The last relation can be written as

$\operatorname{card}\left(\{i+k n ; k \in \mathbb{Z}\} \cap\left[0, d^{\prime}-1\right]\right)=\operatorname{card}\left(\{i+k n ; k \in \mathbb{Z}\} \cap\left[a n+b, a n+b+d^{\prime}-1\right]\right)$.

Therefore, for each $i \in\{0,1, \ldots, n-1\}$

$$
\operatorname{card}\left(\{i+k n ; k \in \mathbb{Z}\} \cap\left[0, d^{\prime}-1\right]\right)=\operatorname{card}\left(\{i+k n ; k \in \mathbb{Z}\} \cap\left[b, b+d^{\prime}-1\right]\right),
$$
i.e.,

$$
\operatorname{card}\left(\{i\} \cap\left[0, d^{\prime}-1\right]\right)=\operatorname{card}\left(\{i+k n ; k \in \mathbb{Z}\} \cap\left[b, b+d^{\prime}-1\right]\right) .
$$

Suppose $b \geq 1$. The preceding relation for $i=d^{\prime}-1$ implies $b \leq d^{\prime}-1$, and further $b<d^{\prime}$. The case $i=d^{\prime}$ implies $d^{\prime}<b$. This is a contradiction. The proof is now complete.

9.3. We also need the following result, a slight modification of [Tadić 1996, Lemma 4.2]. The proof is almost the same. Therefore, we omit it here.

Lemma. Suppose $r=$ an $(a \in \mathbb{Z}, a \geq 1)$ and $n \nmid d$.

(i) $W_{r}^{\prime}(n) X_{r}(n, d) W_{r}^{\prime}(n)=X_{r}(n, d)$.

(ii) $X_{r}(n, d)$ normalizes $W_{r}^{\prime}(n)$.

(iii) For any $w \in X_{r}(n, d)$ we have $X_{r}(n, d)=w W_{r}^{\prime}(n)=W_{r}^{\prime}(n) w$. 
(iv) For each $i \in\{0,1,2, \ldots, r-1\}$ write $i=s(i) n+t(i)$ where $s(i), t(i) \in \mathbb{Z}$ and $0 \leq t(i) \leq n-1$. Let $d=c n+d^{\prime}$, where $c, d^{\prime} \in \mathbb{Z}, 1 \leq d^{\prime} \leq n-1(\leq r-1)$ $\left(d^{\prime} \neq 0\right.$ since $\left.n \nmid d\right)$. Define $w_{(n, d)} \in W_{r}^{\prime}$ by

$$
w_{(n, d)}(i)= \begin{cases}i+\left(n-d^{\prime}\right) & \text { if } t(i) \leq d^{\prime}-1, \\ i-d^{\prime} & \text { if } t(i) \geq d^{\prime} .\end{cases}
$$

Then $w_{(n, d)} \in X_{r}(n, d)$ and $(-1)^{w_{(n, d)}}=(-1)^{(r / n)\left(n-d^{\prime}\right) d^{\prime}}=(-1)^{(r / n)(n-d) d}$.

9.4. As before, let $\Pi=L\left(\left(\left[\rho, v^{d-1} \rho\right]\right.\right.$, $\left.\left.\left[v \rho, v^{d} \rho\right], \ldots,\left[v^{r-1} \rho, v^{r-1+d-1} \rho\right]\right)\right)$.

Recall that we assume in this section that $r \leq d$ and $s_{\rho^{\prime}}=n \nmid d$. To get a nontrivial $\operatorname{LJ}(\Pi)$, we have seen that one needs to consider only the case $s_{\rho^{\prime}}=n \mid r d$. The lemma in $\$ 9.2$ gives further reduction to the case $s_{\rho^{\prime}}=n \mid r$ (see the calculation bellow). This is the reason why we shall assume it in the sequel.

Now we compute in this case

$$
\begin{aligned}
& \mathrm{LJ}(\Pi)=\mathrm{LJ}\left(L\left(\left(\left[\rho, v^{d-1} \rho\right],\left[v \rho, v^{d} \rho\right], \ldots,\left[v^{r-1} \rho, v^{r-1+d-1} \rho\right]\right)\right)\right) \\
& =\sum_{w \in W_{r}^{\prime}}(-1)^{w} \prod_{i=0}^{r-1} \mathrm{LJ}\left(\delta\left(\left[v^{i} \rho, v^{w(i)+(d-1)} \rho\right]\right)\right) \\
& =\sum_{w \in X_{r}(n, d)}(-1)^{w} \prod_{i=0}^{r-1} \mathrm{LJ}\left(\delta\left(\left[v^{i} \rho, v^{w(i)+(d-1)} \rho\right]\right)\right) \\
& =\sum_{w \in w_{(n, d)} W_{r}^{\prime}(n)}(-1)^{w} \prod_{i=0}^{r-1} \mathrm{LJ}\left(\delta\left(\left[v^{i} \rho, v^{w(i)+(d-1)} \rho\right]\right)\right) \\
& =(-1)^{w_{(n, d)}} \sum_{w \in W_{r}^{\prime}(n)}(-1)^{w} \prod_{i=0}^{r-1} \mathrm{LJ}\left(\delta\left(\left[v^{i} \rho, v^{w_{(n, d)} w(i)+(d-1)} \rho\right]\right)\right) \\
& =(-1)^{a(n-d) d} \sum_{w \in W_{r}^{\prime}(n)}(-1)^{w} \prod_{\ell=0}^{n-1} \prod_{j=0}^{a-1} \mathrm{LJ}\left(\delta\left(\left[v^{\ell+n j} \rho, v^{w_{(n, d)} w(\ell+n j)+(d-1)} \rho\right]\right)\right) .
\end{aligned}
$$

Thus

$$
(-1)^{a(n-d) d} \operatorname{LJ}(\Pi)
$$

$$
\begin{aligned}
& =\sum_{\substack{w_{0}^{\prime} \in W_{r}^{\prime}(n ; 0), \ldots, w_{n-1}^{\prime} \in W_{r}^{\prime}(n ; n-1)}} \prod_{\ell=0}^{n-1}(-1)^{w_{\ell}^{\prime}} \prod_{j=0}^{a-1} \mathrm{LJ}\left(\delta\left(\left[v^{\ell+n j} \rho, v^{w_{(n, d)} w_{\ell}^{\prime}(\ell+n j)+(d-1)} \rho\right]\right)\right) \\
& =\sum_{w_{0}, \ldots, w_{n-1} \in W_{a}^{\prime}} \prod_{\ell=0}^{n-1}(-1)^{w_{\ell}} \prod_{j=0}^{a-1} \mathrm{LJ}\left(\delta\left(\left[v^{\ell+n j} \rho, v^{w_{(n, d)}\left(\ell+n w_{\ell}(j)\right)+(d-1)} \rho\right]\right)\right)
\end{aligned}
$$




$$
\begin{aligned}
&= \sum_{w_{0}, \ldots, w_{n-1} \in W_{a}^{\prime}} \prod_{\ell=0}^{d^{\prime}-1}(-1)^{w_{\ell}} \prod_{j=0}^{a-1} \mathrm{LJ}\left(\delta\left(\left[v^{\ell+n j} \rho, v^{w_{(n, d)}\left(\ell+n w_{\ell}(j)\right)+(d-1)} \rho\right]\right)\right) \\
& \times \prod_{\ell=d^{\prime}}^{n-1}(-1)^{w_{\ell}} \prod_{j=0}^{a-1} \mathrm{LJ}\left(\delta\left(\left[v^{\ell+n j} \rho, v^{w_{(n, d)}\left(\ell+n w_{\ell}(j)\right)+(d-1)} \rho\right]\right)\right) \sum_{w_{0}, \ldots, w_{n-1} \in W_{a}^{\prime}} \prod_{\ell=0}^{d^{\prime}-1}(-1)^{w_{\ell}} \prod_{j=0}^{a-1} \mathrm{LJ}\left(\delta\left(\left[v^{\ell+n j} \rho, v^{\ell+n w_{\ell}(j)+\left(n-d^{\prime}\right)+(d-1)} \rho\right]\right)\right) \\
&= \prod_{\ell=0} \sum_{w_{\ell} \in W_{a}^{\prime}}(-1)^{w_{\ell}} \prod_{j=0}^{a-1} \operatorname{LJ}\left(\delta\left(\left[v^{\ell+n j} \rho, v^{\ell+n w_{\ell}(j)+\left(n-d^{\prime}\right)+(d-1)} \rho\right]\right)\right) \\
& \times \prod_{\ell=d^{\prime}}^{n-1} \sum_{w_{\ell} \in W_{a}^{\prime}}(-1)^{w_{\ell}} \prod_{j=0}^{a-1} \operatorname{LJ}\left(\delta\left(\left[v^{\ell+n j} \rho, v^{\ell+n w_{\ell}(j)-d^{\prime}+(d-1)} \rho\right]\right)\right) .
\end{aligned}
$$

We assume in the sequel that $\delta\left(\left[\rho, v^{n-1} \rho\right]\right)$ corresponds to $\rho^{\prime}$ under the JacquetLanglands correspondence as in $\S 6.2$. Then the previous expression becomes

$$
\begin{aligned}
& =\prod_{\ell=0}^{d^{\prime}-1} \sum_{w_{\ell} \in W_{a}^{\prime}}(-1)^{w_{\ell}} \prod_{j=0}^{a-1} \delta\left(\left[v^{\ell+n j} \rho^{\prime}, v^{\ell+n j} v_{\rho^{\prime}}^{w_{\ell}(j)-j+c} \rho^{\prime}\right]\right) \\
& \times \prod_{\ell=d^{\prime}} \sum_{w_{\ell} \in W_{a}^{\prime}}^{n-1}(-1)^{w_{\ell}} \prod_{j=0}^{a-1} \delta\left(\left[v^{\ell+n j} \rho^{\prime}, v^{\ell+n j} v_{\rho^{\prime}}^{w_{\ell}(j)-j+c-1} \rho^{\prime}\right]\right) \\
& =\prod_{\ell=0}^{d^{\prime}-1} v_{\rho^{\prime}}^{\ell / n} \sum_{w_{\ell} \in W_{a}^{\prime}}(-1)^{w_{\ell}} \prod_{j=0}^{a-1} \delta\left(\left[v_{\rho^{\prime}}^{j} \rho^{\prime}, v_{\rho^{\prime}}^{w_{\ell}(j)+c} \rho^{\prime}\right]\right) \\
& =\prod_{\ell=0} v_{\rho^{\prime}}^{\ell / n} L\left(\left(\left[\rho^{\prime}, v_{\rho^{\prime}}^{c} \rho^{\prime}\right], \ldots,\left[v_{\rho^{\prime}}^{a-1} \rho^{\prime}, v_{\rho^{\prime}}^{a-1+c} \rho^{\prime}\right]\right)\right) \\
& \times \prod_{\ell=d^{\prime}}^{n-1} v_{\rho^{\prime}}^{\ell / n} \sum_{w_{\ell} \in W_{a}^{\prime}}(-1)^{w_{\ell}} \prod_{j=0}^{a-1} \delta\left(\left[v_{\rho^{\prime}}^{j} \rho^{\prime}, v_{\rho^{\prime}}^{w_{\ell}(j)+c-1} \rho^{\prime}\right]\right) \\
& \times \nu_{\rho^{\prime}}^{\ell / n} L\left(\left(\left[\rho^{\prime}, v_{\rho^{\prime}}^{c-1} \rho^{\prime}\right], \ldots,\left[v_{\rho^{\prime}}^{a-1} \rho^{\prime}, v_{\rho^{\prime}}^{a-1+c-1} \rho^{\prime}\right]\right)\right)
\end{aligned}
$$

since $a \leq c$, and then clearly $a<c+1$. (Note that we assume $r \leq d$, hence $a n \leq c n+d^{\prime}$, hence $a n<c n$, hence $a<c$; in particular, $a \leq c$.) 
We have proved

$$
\begin{aligned}
& \operatorname{LJ}(\Pi)= \operatorname{LJ}\left(L\left(\left(\left[\rho, v^{d-1} \rho\right], \ldots,\left[v^{r-1} \rho, v^{r-1+d-1} \rho\right]\right)\right)\right) \\
&=(-1)^{a(n-d) d} \prod_{\ell=0}^{d^{\prime}-1} v_{\rho^{\prime}}^{\ell / n} L\left(\left(\left[\rho^{\prime}, v_{\rho^{\prime}}^{c} \rho^{\prime}\right], \ldots,\left[v_{\rho^{\prime}}^{a-1} \rho^{\prime}, v_{\rho^{\prime}}^{a-1+c} \rho^{\prime}\right]\right)\right) \\
& \quad \times \prod_{\ell=d^{\prime}}^{n-1} v_{\rho^{\prime}}^{\ell / n} L\left(\left(\left[\rho^{\prime}, v_{\rho^{\prime}}^{c-1} \rho^{\prime}\right], \ldots,\left[v_{\rho^{\prime}}^{a-1} \rho^{\prime}, v_{\rho^{\prime}}^{a-1+c-1} \rho^{\prime}\right]\right)\right) .
\end{aligned}
$$

9.5. Now suppose that $\rho$ is unitary and that $\rho^{\prime \prime}$ corresponds to $\delta\left(\rho, s_{\rho^{\prime}}\right)=\delta(\rho, n)$ under the Jacquet-Langlands correspondence (as at the beginning of §7.3). Then $\rho^{\prime \prime}$ is unitary and

$$
\rho^{\prime \prime}=\mathrm{LJ}(\delta(\rho, n))=\mathrm{LJ}\left(v^{-(n-1) / 2} \delta\left(\left[\rho, v^{n-1} \rho\right]\right)\right)=v_{\rho^{\prime}}^{-(n-1) /(2 n)} \rho^{\prime},
$$

that is,

$$
\rho^{\prime \prime}=v_{\rho^{\prime}}^{-(n-1) /(2 n)} \rho^{\prime}, \quad \rho^{\prime}=v_{\rho^{\prime}}^{(n-1) /(2 n)} \rho^{\prime \prime}, \quad v_{\rho^{\prime}}=v_{\rho^{\prime \prime}} .
$$

Now (for $n \nmid d$ )

$\mathrm{LJ}\left(L\left(\langle r, d\rangle^{\rho}\right)\right)$

$$
\begin{aligned}
& =\mathrm{LJ}\left(v^{-\frac{r+d}{2}+1} L\left(\left(\left[\rho, v^{d-1} \rho\right], \ldots,\left[v^{r-1} \rho, v^{r-1+d-1} \rho\right]\right)\right)\right) \\
& =(-1)^{a(n-d) d} v^{-\frac{r+d}{2}+1} \prod_{\ell=0}^{d^{\prime}-1} v_{\rho^{\prime}}^{\frac{\ell}{n}} L\left(\left(\left[\rho^{\prime}, v_{\rho^{\prime}}^{c} \rho^{\prime}\right], \ldots,\left[v_{\rho^{\prime}}^{a-1} \rho^{\prime}, v_{\rho^{\prime}}^{a-1+c} \rho^{\prime}\right]\right)\right) \\
& \times \prod_{\ell=d^{\prime}}^{n-1} v_{\rho^{\prime}}^{\frac{\ell}{n}} L\left(\left(\left[\rho^{\prime}, v_{\rho^{\prime}}^{c-1} \rho^{\prime}\right], \ldots,\left[v_{\rho^{\prime}}^{a-1} \rho^{\prime}, v_{\rho^{\prime}}^{a-1+c-1} \rho^{\prime}\right]\right)\right) \\
& =(-1)^{a(n-d) d} v_{\rho^{\prime}}^{-\frac{a+c+d^{\prime} / n}{2}+\frac{1}{n}} \prod_{\ell=0}^{d^{\prime}-1} v_{\rho^{\prime}}^{\frac{\ell}{n}} v_{\rho^{\prime}}^{\frac{a+c+1}{2}-1} L\left(\langle a, c+1\rangle^{\rho^{\prime}}\right) \\
& \times \prod_{\ell=d^{\prime}}^{n-1} v_{\rho^{\prime}}^{\frac{\ell}{n}} v_{\rho^{\prime}}^{\frac{a+c}{2}-1} L\left(\langle a, c\rangle^{\rho^{\prime}}\right) \\
& =(-1)^{a(n-d) d} \prod_{\ell=0}^{d^{\prime}-1} v_{\rho^{\prime}}^{\frac{2 \ell-n-d^{\prime}+2}{2 n}} L\left(\langle a, c+1\rangle^{\rho^{\prime}}\right) \prod_{\ell=d^{\prime}}^{n-1} v_{\rho^{\prime}}^{\frac{2 \ell-2 n-d^{\prime}+2}{2 n}} L\left(\langle a, c\rangle^{\rho^{\prime}}\right) \\
& =(-1)^{a(n-d) d} \prod_{\ell=0}^{d^{\prime}-1} v_{\rho^{\prime}}^{\frac{2 \ell-n-d^{\prime}+2}{2 n}} L\left(\langle a, c+1\rangle^{v^{v^{\prime}}} \rho^{\frac{n-1}{2 n}} \rho^{\prime \prime}\right) \prod_{\ell=d^{\prime}}^{n-1} v_{\rho^{\prime}}^{\frac{2 \ell-2 n-d^{\prime}+2}{2 n}} L\left(\langle a, c\rangle^{v_{\rho^{\prime}}^{\frac{n-1}{2 n}} \rho^{\prime \prime}}\right) \\
& =(-1)^{a(n-d) d} \prod_{\ell=0}^{d^{\prime}-1} v_{\rho^{\prime \prime}}^{\frac{2 \ell-d^{\prime}+1}{2 n}} L\left(\langle a, c+1\rangle^{\rho^{\rho^{\prime \prime}}}\right) \prod_{\ell=d^{\prime}}^{n-1} v_{\rho^{\prime \prime}}^{\frac{2 \ell-n-d^{\prime}+1}{2 n}} L\left(\langle a, c\rangle^{\rho^{\prime \prime}}\right)
\end{aligned}
$$




$$
=(-1)^{a(n-d) d} \prod_{\ell=-\frac{d^{\prime}-1}{2}}^{\frac{d^{\prime}-1}{2}} v_{\rho^{\prime \prime}}^{\frac{\ell}{n}} L\left(\langle a, c+1\rangle^{\rho^{\prime \prime}}\right) \prod_{\ell=d^{\prime}}^{n-1} v_{\rho^{\prime \prime}}^{\frac{\left(-n-d^{\prime}+1\right) / 2+\ell}{n}} L\left(\langle a, c\rangle^{\rho^{\prime \prime}}\right) .
$$

Therefore, we have proved:

Proposition. Suppose that (U0) holds. Assume $n \nmid d, n \mid r$, and $r \leq d$. Write $d=$ $c n+d^{\prime}$, with $c, d \in \mathbb{Z}$ and $1 \leq d^{\prime} \leq n-1$. Then

\section{$\operatorname{LJ}\left(L\left(\langle r, d\rangle^{\rho}\right)\right)$}

$$
\begin{aligned}
& =(-1)^{a(n-d) d} \operatorname{str}_{v_{\rho^{\prime \prime}}}\left(d^{\prime}, n, L\left(\langle r / n, c+1\rangle^{\rho^{\prime \prime}}\right)\right) \operatorname{str}_{v_{\rho^{\prime \prime}}}\left(n-d^{\prime}, n, L\left(\langle r / n, c\rangle^{\rho^{\prime \prime}}\right)\right) \\
& =(-1)^{a(n-d) d} \operatorname{str}\left(d^{\prime}, L\left(\langle r / n, c+1\rangle^{\rho^{\prime \prime}}\right)\right) \operatorname{str}\left(n-d^{\prime}, L\left(\langle r / n, c\rangle^{\rho^{\prime \prime}}\right)\right)
\end{aligned}
$$

This expression can be also written as

$$
\begin{aligned}
(-1)^{a(n-d) d} \operatorname{str}(d-[d / n] n, L(\langle r / n,[ & \left.\left.d / n]+1\rangle^{\rho^{\prime \prime}}\right)\right) \\
& \times \operatorname{str}\left(([d / n]+1) n-d, L\left(\langle r / n,[d / n]\rangle^{\rho^{\prime \prime}}\right)\right) .
\end{aligned}
$$

\section{Unitarity of Jacquet-Langlands correspondence of irreducible unitary representations, II}

Under the assumptions of the previous section, we first show in this section that $\mathrm{LJ}\left(L\left(\langle r, d\rangle^{\rho}\right)\right)$ is unitary (we assume $\rho$ to be unitary). Then we show that

$$
\mathrm{LJ}\left(v^{\beta}\left(L\left(\langle r, d\rangle^{\rho}\right)\right) \times v^{-\beta}\left(L\left(\langle r, d\rangle^{\rho}\right)\right)\right)
$$

is unitary for $0<\beta<\frac{1}{2}$ (under the same assumptions).

For the unitarity of $\operatorname{LJ}\left(L\left(\langle r, d\rangle^{\rho}\right)\right)$, it is enough to show that

$$
0 \leq \frac{d^{\prime}-1}{2 n}<\frac{1}{2} \quad \text { and } \quad 0 \leq \frac{n-d^{\prime}-1}{2 n}<\frac{1}{2} .
$$

Both obviously hold (recall $1 \leq d^{\prime} \leq n-1$ ).

For the complementary series, we need to see that

$$
0 \leq \frac{d^{\prime}-1}{2 n}+\frac{\beta}{n}<\frac{1}{2} \quad \text { and } \quad 0 \leq \frac{n-d^{\prime}-1}{2 n}+\frac{\beta}{n}<\frac{1}{2}
$$

for $0<\beta<\frac{1}{2}$ (see Section 9). This holds since $1 \leq d^{\prime} \leq n-1$ and $\beta<\frac{1}{2}$.

\section{Jacquet-Langlands correspondence in the unitary case: the remaining cases}

It remains to compute $\operatorname{LJ}\left(L\left(\langle d, r\rangle^{\rho}\right)\right)$ in the case $d \geq r$. Since $\mathrm{LJ}$ and ${ }^{t}$ commute up to a sign (see [Badulescu 2002, Theorem 3.17]), we have

$$
\mathrm{LJ}\left(L\left(\langle d, r\rangle^{\rho}\right)\right)=\mathrm{LJ}\left(L\left(\langle r, d\rangle^{\rho}\right)^{t}\right)= \pm \mathrm{LJ}\left(L\left(\langle r, d\rangle^{\rho}\right)\right)^{t} .
$$


Therefore, we can apply our previous calculations. We have two cases.

- If $r \leq d, n \mid d, r=a n+b, a, b \in \mathbb{Z}, 0 \leq b \leq n-1,1 \leq n$, then

$$
\begin{aligned}
\operatorname{LJ}\left(L\left(\langle d, r\rangle^{\rho}\right)\right) & = \pm \operatorname{LJ}\left(L\left(\langle r, d\rangle^{\rho}\right)\right)^{t} \\
& = \pm \operatorname{str}\left(b, L\left(\langle d / n, a+1\rangle^{\rho^{\prime \prime}}\right)\right) \operatorname{str}\left(\min (n, r)-b, L\left(\langle d / n, a\rangle^{\rho^{\prime \prime}}\right)\right) .
\end{aligned}
$$

- If $n \nmid d, n \mid r, r \leq d, d=c n+d^{\prime}, c, d \in \mathbb{Z}, 1 \leq d^{\prime} \leq n-1$, then

$$
\begin{aligned}
\operatorname{LJ}\left(L\left(\langle d, r\rangle^{\rho}\right)\right) & = \pm \operatorname{LJ}\left(L\left(\langle r, d\rangle^{\rho}\right)\right)^{t} \\
& = \pm(-1)^{a(n-d) d} \operatorname{str}\left(d^{\prime}, L\left(\langle c+1, r / n\rangle^{\rho^{\prime \prime}}\right)\right) \operatorname{str}\left(n-d^{\prime}, L\left(\langle c, r / n\rangle^{\rho^{\prime \prime}}\right)\right) .
\end{aligned}
$$

Here too unitarity is preserved, by the results in Sections 8 and 10, since the involution preserves unitarity. (We assume as before that (U0) holds.)

One can compute the sign in the two preceding formulas using [Badulescu 2002, Theorem 3.17]. (Our involutions differ by a sign from the ones used there.)

\section{Acknowledgements}

A part of this work was done during our visit to the Institute for Mathematical Sciences of the National University of Singapore, for the special period on the representation theory of Lie groups. The work was finished during our visit to Université Paris 7 - Denis Diderot. We are thankful to both institutions for their hospitality. We are also thankful to the referee and to A. I. Badulescu for a number of suggestions that helped improve the paper.

\section{References}

[Aubert 1995] A.-M. Aubert, "Dualité dans le groupe de Grothendieck de la catégorie des représentations lisses de longueur finie d'un groupe réductif p-adique", Trans. Amer. Math. Soc. 347:6 (1995), 2179-2189. Erratum in 348:11 (1996), 4687-4690. MR 95i:22025 Zbl 0827.22005

[Badulescu 2002] A. I. Badulescu, "Correspondance de Jacquet-Langlands étendue à toutes les représentations", preprint, 2002. math.GR/0201117

[Badulescu and Renard 2004] A. I. Badulescu and D. A. Renard, "Sur une conjecture de Tadić", Glas. Mat. Ser. III 39(59):1 (2004), 49-54. MR 2005d:22016 Zbl 1060.22015

[Bernstein 1984] J. N. Bernstein, " $P$-invariant distributions on $\operatorname{GL}(N)$ and the classification of unitary representations of GL( $N)$ (non-Archimedean case)", pp. 50-102 in Lie group representations (College Park, MD, 1982/1983), vol. II, edited by R. Herb et al., Lecture Notes in Math. 1041, Springer, Berlin, 1984. MR 86b:22028 Zbl 0541.22009

[Deligne et al. 1984] P. Deligne, D. Kazhdan, and M.-F. Vignéras, "Représentations des algèbres centrales simples $p$-adiques", pp. 33-117 in Représentations des groupes réductifs sur un corps local, Travaux en Cours, Hermann, Paris, 1984. MR 86h:11044 Zbl 0583.22009

[Jacquet and Langlands 1970] H. Jacquet and R. P. Langlands, Automorphic forms on GL(2), Lecture Notes in Math. 114, Springer, Berlin, 1970. MR 53 \#5481 Zbl 0236.12010 
[Knapp and Rogawski 1997] A. W. Knapp and J. D. Rogawski, "Applications of the trace formula", pp. 413-431 in Representation theory and automorphic forms (Edinburgh, 1996), edited by T. N. Bailey and A. W. Knapp, Proc. Sympos. Pure Math. 61, Amer. Math. Soc., Providence, RI, 1997. MR 98j:11105 Zbl 01099109

[Langlands 1970] R. P. Langlands, "Problems in the theory of automorphic forms", pp. 18-61 in Lectures in modern analysis and applications, III, edited by C. T. Taam, Lecture Notes in Math. 170, Springer, Berlin, 1970. MR 46 \#1758 Zbl 0225.14022

[Miličić 1973] D. Miličić, "On $C^{*}$-algebras with bounded trace”, Glasnik Mat. Ser. III 8 (28) (1973), 7-22. MR 48 \#2781 Zbl 0265.46072

[Rodier 1982] F. Rodier, "Représentations de GL $(n, k)$ où $k$ est un corps $p$-adique", pp. 201-218 (exposé 587) in Séminaire Bourbaki, 1981/1982, Astérisque 92-93, Soc. Math. France, Paris, 1982. MR 84h:22040 Zbl 0506.22019

[Rogawski 1983] J. D. Rogawski, "Representations of GL( $n)$ and division algebras over a $p$-adic field”, Duke Math. J. 50:1 (1983), 161-196. MR 84j:12018 Zbl 0523.22015

[Schneider and Stuhler 1997] P. Schneider and U. Stuhler, "Representation theory and sheaves on the Bruhat-Tits building”, Inst. Hautes Études Sci. Publ. Math. 85 (1997), 97-191. MR 98m:22023 Zbl 0892.22012

[Tadić 1986] M. Tadić, "Classification of unitary representations in irreducible representations of general linear group (non-Archimedean case)", Ann. Sci. École Norm. Sup. (4) 19:3 (1986), 335382. MR 88b:22021 Zbl 0614.22005

[Tadić 1987] M. Tadić, “Topology of unitary dual of non-Archimedean GL(n)”, Duke Math. J. 55:2 (1987), 385-422. MR 89c:22029 Zbl 0668.22006

[Tadić 1988] M. Tadić, "On limits of characters of irreducible unitary representations", Glas. Mat. Ser. III 23 (43): 1 (1988), 15-25. MR 90b:22019 Zbl 0655.22003

[Tadić 1990] M. Tadić, "Induced representations of $\operatorname{GL}(n, A)$ for $p$-adic division algebras $A$ ", $J$. Reine Angew. Math. 405 (1990), 48-77. MR 91i:22025 Zbl 0684.22008

[Tadić 1993] M. Tadić, "An external approach to unitary representations", Bull. Amer. Math. Soc. (N.S.) 28:2 (1993), 215-252. MR 93g:22020 Zbl 0799.22010

[Tadić 1995] M. Tadić, "On characters of irreducible unitary representations of general linear groups", Abh. Math. Sem. Univ. Hamburg 65 (1995), 341-363. MR 96m:22039 Zbl 0856.22026

[Tadić 1996] M. Tadić, "Correspondence on characters of irreducible unitary representations of GL(n, C)", Math. Ann. 305:3 (1996), 419-438. MR 97f:22026 Zbl 0854.22020

[Vogan 1986] D. A. Vogan, Jr., "The unitary dual of GL(n) over an Archimedean field", Invent. Math. 83:3 (1986), 449-505. MR 87i:22042 Zbl 0598.22008

[Zelevinsky 1980] A. V. Zelevinsky, "Induced representations of reductive p-adic groups, II: On irreducible representations of GL(n)”, Ann. Sci. École Norm. Sup. (4) 13:2 (1980), 165-210. MR 83g: 22012 Zbl 0441.22014

Received March 19, 2004.

\section{MARKO TADIĆ}

DEPARTMENT OF MATHEMATICS

UNIVERSITY OF ZAGREB

BIJENIČKA 30

10000 ZAGREB

CROATIA

tadic@math.hr 